\title{
A jet-dominated model for a broad-band spectral energy distribution of the nearby low-luminosity active galactic nucleus in M94
}

\author{
Pieter van Oers ${ }^{1}$, Sera Markoff ${ }^{1}$, Phil Uttley ${ }^{1}$, Ian $\mathrm{McHardy}^{2}$, \\ Tessel van der Laan ${ }^{3}$, Jennifer Donovan Meyer ${ }^{4}$, Riley Connors ${ }^{1}$ \\ ${ }_{1}^{1}$ Anton Pannekoek Institute for Astronomy, University of Amsterdam, Science Park 904, 1098 XH, Amsterdam, The Netherlands \\ ${ }^{2}$ School of Physics and Astronomy, University of Southampton, Southampton, S017 1BJ, UK \\ ${ }^{3}$ Institut de Radioastronomie Millimétrique, 300 rue de la Piscine, 38406 St. Martin d'Hères, Grenoble, France \\ ${ }^{4}$ National Radio Astronomy Observatory, Charlottesville, VA 22901, USA
}

20 April 2017

\begin{abstract}
We have compiled a new multi-wavelength spectral energy distribution (SED) for the closest obscured low ionisation emission line region (LINER) active galactic nucleus (AGN), NGC 4736, also known as M94. The SED comprises mainly high resolution (mostly sub-arcsecond, or, at the distance to M94, 23 pc from the nucleus) observations from the literature, archival data, as well as previously unpublished submillimetre data from the Plateau de Bure Interferometer (PdBI) and the Combined Array for Research in Millimeter-wave Astronomy (CARMA), in conjunction with new electronic Multi Element Radio Interferometric Network (e-MERLIN) L-band $(1.5 \mathrm{GHz})$ observations. Thanks to the e-MERLIN resolution and sensitivity we resolve for the first time a double structure composed of two radio sources separated by $\sim 1^{\prime \prime}$, previously observed only at higher frequency. We explore this dataset, that further includes non-simultaneous data from the Very Large Array (VLA), the Gemini telescope, the Hubble Space Telescope $(H S T)$ and the Chandra X-ray observatory, in terms of an outflow-dominated model. We compare our results with previous trends found for other AGN using the same model (NGC 4051, M81*, M87 and Sgr A*), as well as hard and quiescent state X-ray binaries. We find that the nuclear broadband spectrum of M94 is consistent with a relativistic outflow of low inclination. The findings in this work add to the growing body of evidence that the physics of weakly accreting black holes scales with mass in a rather straightforward fashion.
\end{abstract}

Key words: Black hole physics - accretion, accretion discs - radiation mechanisms: non-thermal - galaxies: active - galaxies: jets - radio continuum: galaxies

\section{INTRODUCTION}

At $4.8 \pm 0.8 \mathrm{Mpc}, \mathrm{M} 94$ is the closest known galaxy that has a low-luminosity active galactic nucleus (LLAGN) classified as an obscured low ionisation emission line region LINER (L2, Ho et al. 1997; Roberts et al. 2001) and is the least luminous member of the LINER class found thus far $\left(L_{\mathrm{bol}}\right.$ $\sim 2.5 \times 10^{40} \mathrm{erg} \mathrm{s}^{-1}$; Constantin \& Seth 2012). Therefore the M94 nucleus represents a unique possibility to probe the extremely low-accretion rate physics thought to govern these types of sources. In this work we aim to do a multiwavelength study of this source and hence we will first briefly describe this source in different wavebands.
M94 has an optical radius $R_{25}$ of $\sim 5.6^{\prime}$ or $\sim 7 \mathrm{kpc}$ (Trujillo et al. 2009) and was identified as a member of Hubble class (R)SAB(rs)ab (Hubble 1926; Buta et al. 2007). i.e. an early type spiral galaxy with star-forming rings. The central surface brightness profile in infrared reveals a double Sérsic structure (Richings et al. 2011, these sources are on average less radio-loud than so-called "core" galaxies). Eracleous et al. (2002) observed a complex X-ray structure, harbouring a myriad of discrete X-ray sources.

Maoz et al. (1995, 2005) speculate that the galaxy hosts a double AGN in the process of merging, which may have triggered a starburst episode and could explain the observed 
morphological and kinematic features. On the other hand, there has been much debate about whether even a single AGN is lurking in M94, as the LINER could just as easily be ionised by e.g. the UV radiation from hot, young stars, collisional shocks or a population of stellar mass black holes (BHs; e.g. Roberts et al. 1999; Pellegrini et al. 2002 and references there-in). However evidence that M94 harbours an AGN has been accumulating: The second brightest Xray source (as seen by Chandra) has counterparts in the UV (Maoz et al. 2005) and radio (Körding et al. 2005; Nagar et al. 2002, 2005) and hence seems to correspond to a supermassive BH (SMBH). Further evidence for the AGN nature of this source includes e.g. its variability in the UV (Maoz et al. 2005), and more recently the analysis of archival $H S T$ Space Telescope Imaging Spectrograph (STIS) data by Constantin \& Seth (2012), who discovered a broad H $\alpha$ emission line in the nuclear optical spectrum; generally considered the "smoking gun" for AGN emission.

Perhaps the best evidence for what is the dominant source of ionisation in M94 comes from detailed optical spectroscopic analysis of the emission lines originating from the narrow line region (NLR). Emission line diagnostic diagrams (generally referred to as BPT diagrams, after Baldwin et al. 1981) make use of the ratios of optical lines $([\mathrm{N} \mathrm{II}] / \mathrm{H} \alpha$, $[\mathrm{O} \mathrm{III}] / \mathrm{H} \beta)$ to separate galaxies dominated by star formation (SF) from those dominated by AGN emission (also see Veilleux \& Osterbrock 1987; Kauffmann et al. 2003; Kewley et al. 2006; Schawinski et al. 2007). When the [S II] and [O I] lines are also detected, the BPT diagrams allow us to accurately classify the cores of galaxies that are not dominated by stellar processes into two main types of AGN: LINERs and Seyferts. The former are generally of lower bolometric luminosity $\left(L_{\mathrm{bol}}\right)$ and higher radio loudness, while the latter are historically associated with higher $L_{\text {bol }}$, radio quiet sources (but see e.g. Ulvestad \& Wilson 1989; Ho \& Peng 2001; Maitra et al. 2011 and references therein). Figure 2 in Constantin \& Seth (2012) summarises the currently available M94 nuclear optical spectroscopy and shows it is consistent with a LINER (also see Moustakas \& Kennicutt 2006).

It is a topic of ongoing investigation whether the two different types of AGN mentioned above may actually correspond or "map" to certain states found in their low-mass relatives, the $\mathrm{BHs}$ in $\mathrm{X}$-ray binaries (i.e. $\mathrm{BH}$ binaries; BHBs) (Merloni et al. 2003; Falcke et al. 2004; Körding et al. 2006; McHardy et al. 2006; Plotkin et al. 2012). We observe BHBs in predominantly two spectral states, named after their appearance in X-rays (see, e.g. Remillard \& McClintock 2006 or Belloni 2010 for reviews on BHB spectral states). The soft state X-ray spectrum is dominated by a soft, thermal component, usually associated with a geometrically thin, optically thick accretion disk (Novikov \& Thorne 1973; Shakura \& Sunyaev 1973). The Hard State (HS) is dominated by an optically thin, hard X-ray component, that is usually thought to be due to inverse Comptonisation of a thermal input (Thorne \& Price 1975; Sunyaev \& Truemper 1979). In addition to this power-law-like emission, the HS also displays high RMS noise and the presence of compact, steady jets. Hence on the surface it is tempting to associate the LINER class with the HS and the Seyfert class with the soft state of BHBs however see Sec. 6.1), but to see how far this ostensible comparison would hold or fail, more quantitative comparisons are necessary.
While BHBs can typically change state on humanly observable timescales of days to weeks, their supermassive counterparts are much slower to go through their evolution because of the length scales involved, with typical timescales expected for state changes easily exceeding $10^{4}$ years (e.g. Keel et al. 2012, 2015; Schawinski et al. 2015 and references therein). Hence it is nearly impossible to directly observe state changes in AGN. Interestingly, however, AGN that show significant variation in the intrinsic luminosity have been observed (see e.g. LaMassa et al. 2015; Koay et al. 2016, and references there-in). In addition, for the first time a quasar was found to "switch off", possibly due to a transition into a radiatively inefficient state (Schawinski et al. 2010). More indirect evidence that AGN can change state comes from X-ray images of cavities in clusters of galaxies, showing activity cycles of $\sim 10^{7}-10^{8}$ years (e.g. Bîrzan et al. 2004). Clearly such findings show the merits of studying and comparing the evolution of BHs on both ends of the massscale, specifically for the predictive nature of low mass BHB evolution when used to infer AGN evolution.

Low accretion rate BHBs are generally thought to harbour a radiatively inefficient accretion flow (RIAF). In such flows a thermal-dominated "cloud" of electrons comprise the inner flow. This cloud can be seen as the inner accretion disk that evaporated into a "corona" (Esin et al. 1997; Gilfanov 2010), or can be interpreted as the base of a jet (Markoff et al. 2005, hereafter MNW05), i.e. a magnetised, outflowing corona (e.g. Beloborodov 1999; Malzac et al. 2001; Merloni \& Fabian 2002). In this work we explore the concept of mass-scaling in BH systems, investigating a newly compiled broadband Spectral Energy Distribution (SED) of the M94 nucleus in the context of an outflow-dominated relativistic jet paradigm (MNW05, see Section 4 for further references). The SED consists of some of the first data acquired with the recently upgraded e-MERLIN, supplemented by previously unpublished Plateau de Bure Interferometer (PdBI) and Combined Array for Research in Millimeter-wave Astronomy (CARMA) continuum data, as well as archival data and data from the literature. The MNW05 framework is, to date, the only model that has been applied successfully to a variety of $\mathrm{BH}$ broadband SEDs on both ends of the mass scale, as well as across the luminosity scale (in terms of the Eddington luminosity, $L_{\mathrm{bol}} / L_{\mathrm{Edd}}$, where $L_{\mathrm{Edd}}=1.26 \times 10^{38}$ $\left[M_{\mathrm{BH}} / \mathrm{M}_{\odot}\right] \mathrm{erg} \mathrm{s}^{-1}$, with $M_{\mathrm{BH}}$ the $\mathrm{BH}$ mass). Specifically the observations investigated with MNW05 range from a multitude of HS and quiescent state BHBs (MNW05; Gallo et al. 2007; Migliari et al. 2007; Maitra et al. 2009a; Plotkin et al. 2015), to the GRS $1915+105$ specific (HS equivalent) $\chi$ state (van Oers et al. 2010) to several SMBHs (Markoff et al. 2007, 2008; Maitra et al. 2009b, 2011; Prieto et al. 2016; Connors et al. 2016, in prep.). Consistent with nomenclature used in some of these works we will refer to the core of M94 specifically, as M94*.

We compare the results from fitting the M94* SED with those previously obtained for BHBs and AGN. The BHBs span a wider range in jet properties and we will limit our comparison to the ranges previously found for the different spectral states. The AGN, however, merit an individual comparison: M94* is the closest "pure" LINER and has a dynamical mass of $6.77 \pm 1.54 \times 10^{6} \mathrm{M}_{\odot}$ (Kormendy \& Ho 2013). M87 is also a type 2 LINER (Ho et al. 1997) and has a similar bolometric luminosity in Eddington. M81 has both LINER 


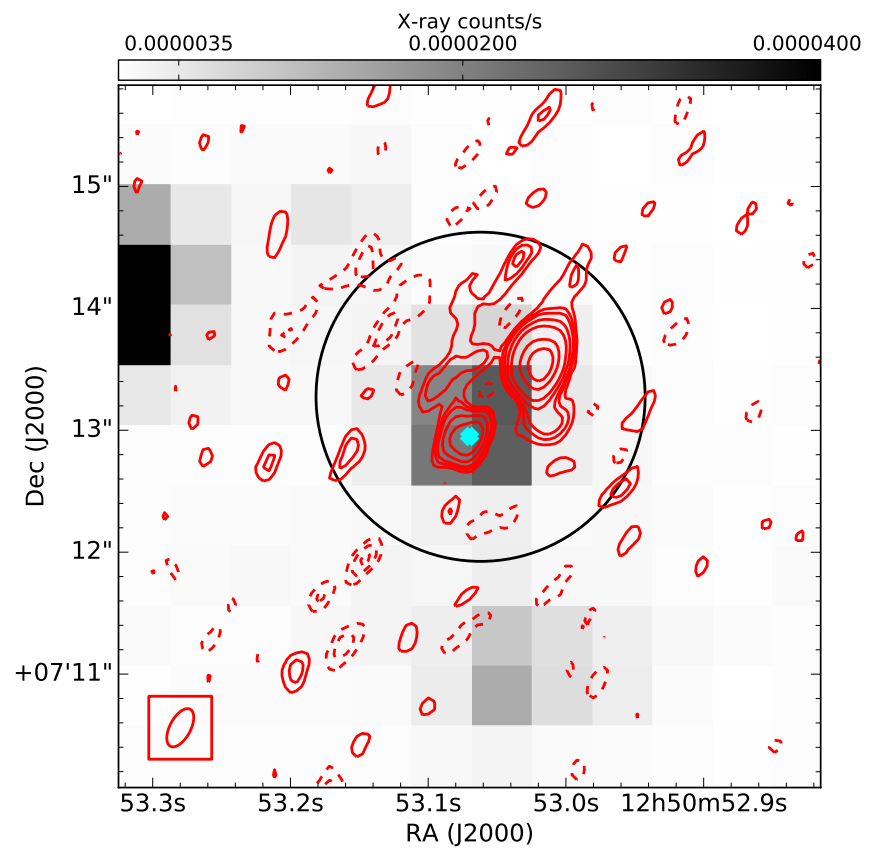

Figure 1. e-MERLIN M94 L-band contour map (in red) overlaid on the Chandra data from 2000 (greyscale; see Section 3.5). The black circle denotes the region used to extract the Chandra spectrum, while the cyan dot denotes the location of the M94* flat-spectrum nucleus at $15 \mathrm{GHz}$ (Nagar et al. 2005). Radio contour levels are at $-7,-5,-3,3,5,7,12,20,45$ and $70 \sigma$, where $\sigma=21 \mu \mathrm{Jy}$ (the latter two contour levels are only relevant for the extended source towards the north west). Dashed contours indicate negative levels. As there are negative levels down to $\sim-5 \sigma$, also levels up to $\sim+5 \sigma$ should be regarded with caution. Hence considering contours of greater than $7 \sigma$ only, M94* appears unresolved.

and Seyfert characteristics, while the narrow line Seyfert 1 (NLS1) NGC 4051 is a poster child for Seyfert galaxies; it is one of the original six studied by Seyfert (1943). Due to its relative proximity $\mathrm{Sgr}$ A* is not classifiable as LINER or Seyfert, but it displays extremely low Eddington luminosities $\left(L_{\mathrm{bol}} / L_{\mathrm{Edd}} \sim 2 \times 10^{-6}\right)$ and at $4 \times 10^{6} M_{\odot}$ (Gillessen et al. $2009)$ its dynamical mass is very similar to that of M94*.

We introduce the new M94* e-MERLIN observations in Section 2 and explain which other data we add to these radio observations in Section 3. Details about the MNW05 framework are in Section 4, while the best-fit models are presented in Section 5. We discuss these results in Section 6 and end with our conclusions in Section 7.

\section{NEW e-MERLIN AND ARCHIVAL VLA OBSERVATIONS}

In this section we present a dataset obtained by the recently upgraded e-MERLIN array. These upgrades have enabled a dramatically increased bandwidth coverage, compared to the original MERLIN configuration (e.g. Garrington et al. 2004). These L-band ( 1.5 GHz) observations are part of the Legacy e-MERLIN Multi-band Imaging of Nearby Galaxies
Survey (LeMMINGS ${ }^{1}$ ). Details about the reduction process are in Section 2.1. This is the first time the double radio structure in M94 (seen at $8.49 \mathrm{GHz}$ VLA; Körding et al. 2005 , but also at $4.9 \mathrm{GHz}$, see below) has been resolved into individual sources at low frequencies (see Figure 1). The weaker of the two (at $1.5 \mathrm{GHz}$ ), towards the south-east, is unresolved within the noise and should therefore correspond to the nucleus, while the other source is extended and of unknown origin. We will investigate the nature of these two sources in more detail below.

\section{1 e-MERLIN radio reduction}

All data inspection, flagging, calibration and imaging is done using the Astronomical Image Processing System (AIPS; Greisen 2003), following the e-MERLIN cookbook $^{2}$. M94 was observed by all 7 e-MERLIN stations (Lovell, Jodrell Mk2, Knockin, Defford, Pickmere, Darham and Cambridge) in L-band and in spectral line observing mode on May 5th and May 7th 2014. The phase calibrator is J1242+3751, the bright point source calibrator is $1407+284$ (or OQ 208) and the absolute flux density calibrator is $1331+305$ (3C 286). The array observed the target and phase calibrator alternately at a $\sim 7 \mathrm{~min} / \sim 3 \mathrm{~min}$ cycle, respectively. The time on source totals $\sim 13.7$ hours. The full bandwidth was 512 $\mathrm{MHz}$, split into 8 spectral windows (SPW)s with each SPW split into 512 channels.

On the 5th of May no flux calibrator was observed, so all fluxes are calibrated using the $1331+305$ observation of May 7th. This is usual practice with e-MERLIN and does not add significant uncertainty, as long as there are no changes in the instrumental system.

Before imaging (with IMAGR), the target visibilities were re-weighted according to individual antenna sensitivity. The calibrated data were imaged with natural weighting to maximise sensitivity. Some iterations of self-calibration were attempted, but with a target flux below $1 \mathrm{mJy}$ and an image dynamic range (DR) of only $\sim 200$, the self-calibration did not lead to improvements. Multi-scale cleaning was attempted, but did not yield an improvement in noise level or DR.

We use separate, source-free noise images, created using a shift in right ascension of $60^{\prime \prime}$, to get an estimate of the RMS of the target image, rather than estimating the RMS from the target image itself. Using this method we can uniformly obtain reasonable estimates of the noise in any target image we make (see Section 2.2), in a way that is less sensitive to local side-lobes ${ }^{3}$ whose exact locus can vary from SPW to SPW. We note that despite the advantages of using an offset noise image, it may sometimes lead to an under-estimate of the true RMS, as it may fail to account for RMS contributions of the local side-lobes mentioned above. However, any such additional error is more systematic than statistical, and hence we prefer to use the errors obtained from the noise images for fitting purposes.

\footnotetext{
1 www.jb.man.ac.uk/ rbeswick/LeMMINGS/

2 www.e-merlin.ac.uk/data_red/tools/e-merlincookbook_V3.0_Feb2015.pdf

3 Such side-lobes can appear due to bad data that has gone unnoticed during flagging and/or the lack of self-calibration.
} 


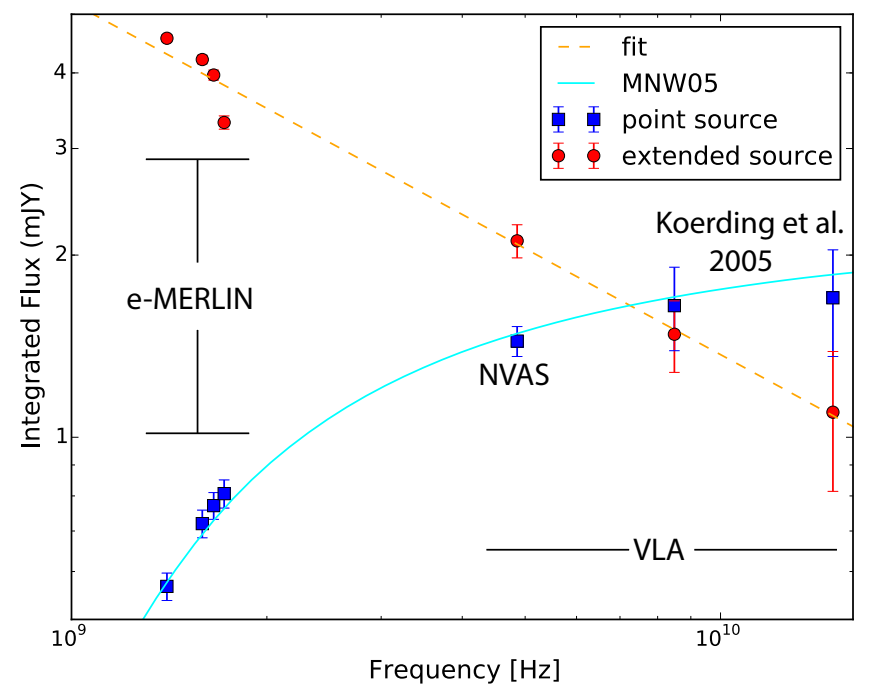

Figure 2. M94 1.5-15 GHz radio spectrum for the point- and extended sources in Figure 1. The origin of these data are summarised in Table 1. The (unresolved) point source spectrum is shown with a jet model running through, while a power-law fit is applied to the (extended) non-core spectrum, best fit with a spectral index $\alpha=0.58$, where $S_{v} \propto v^{-\alpha}$.

Using 100000 iterations for the cleaning process yielded images that portray the lowest RMS of $\sim 20 \mu \mathrm{Jy} / \mathrm{bm}$. Corresponding example radio contours are shown in Figure 1.

\subsection{Extended steep-spectrum radio source}

Since this is the first time the double radio structure in M94 has been resolved in L-band and since the two components differ slightly in coordinates with those found by Körding et al. (2005) it is prudent to investigate the two components, identify the nucleus, and ascertain the nature of the other component. To this end we take advantage of the e-MERLIN broadband capabilities and compile a detailed radio spectrum. Imaging all 8 available SPWs individually however revealed a poor data quality at the lowest-energy SPWs, due to RFI and possibly intrinsic absorption. Hence we bin together the 5 lowest energy SPWs but use individual images for the top three SPWs (cf. Figure A.1). We obtain integrated fluxes for both the point and extended source using AIPS/JMFIT. JMFIT reports the error on the fit, and these errors were added in quadrature to the RMS level of the separate noise images. The results are in Table 1, and plotted in Figure 2.

In addition to the e-MERLIN observations, we have populated the radio SED with other VLA (A-configuration only) high-resolution $\mathrm{C}, \mathrm{X}, \mathrm{U}$ and $\mathrm{K}$ band data points (at $4.9,8.5,15$ and $22 \mathrm{GHz}$ respectively).

The $\mathrm{C}$ and $\mathrm{K}$ band observations were obtained as prereduced images from the automated VLA pipeline NVAS (NRAO VLA Archive Survey) ${ }^{4}$. The C band images, even though dated as far back as 1983 and 1985, already show the double structure mentioned above (however we found no

4 http://archive.nrao.edu/nvas/ publication regarding this finding). M94* is not detected at $\mathrm{K}$ band in A-configuration, due to an RMS of $0.718 \mathrm{mJy}$ that we will use to include a $3 \sigma$ upper limit in the broadband SED (Figure 3). As this $22 \mathrm{GHz}$ data offers no extra information regarding the double structure we ignore it in figure 2 .

To improve $\mathrm{S} / \mathrm{N}$ we average the five $8.49 \mathrm{GHz}$, Aconfiguration measurements from Körding et al. (2005). The observations were done over a 3 month period and although the absolute flux densities reported for both the point and extended source show some variation, within the errors both the flux densities remained essentially constant.

The $15 \mathrm{GHz}$ measurement is reported in Nagar et al. (2002, 2005). These papers do not report a double structure, however at $0.34 \mathrm{mJy}$ (Körding et al. 2005) the image RMS would have prevented them from detecting the extended source at a $5 \sigma$ level. Körding et al. (2005) rereduced the original data and confirmed the extended source at a $4 \sigma$ level, but accurate flux determination from this data remained impossible due to the high RMS. Hence they obtained new $\mathrm{U}$ band maps from which they report both peak and integrated nuclear flux densities of $1.7 \mathrm{mJy}$, matching the integrated flux in the Nagar et al. works. Koerding et al. also report a $1.1 \mathrm{mJy}$ peak flux density for the extended source (and note that the $0.95 \mathrm{mJy}$ integrated flux density probably has some flux missing due to the small integration box used for this source). Unfortunately they report neither errors on these fluxes nor the RMS for the new images, even though it is clear from context that the new $U$ band images are less noisy than the Nagar et al. maps. Hence considering the extra uncertainty due to the "missing" integrated extended source flux and to remain conservative for the point source flux we will use the Nagar et al. RMS of $0.34 \mathrm{mJy}$ as a $1 \sigma$ error on the Koerding et al. $15 \mathrm{GHz}$ fluxes in Figure 2.

Comparing the spectral indices $\left(\alpha\right.$; where $\left.S_{v} \propto v^{-\alpha}\right)$ in Figure 2, we find that the point source in the south east has an inverted spectrum and hence unambiguously identify this source with the core. With $\alpha \propto 0.58$ the northwestern extended component appears to show steep spectrum emission, consistent with slopes seen in star forming regions $(0.5<\alpha<1.0$; e.g. Condon 1992; Dopita et al. 2005; Clemens et al. 2008), or injection indices in extended synchrotron knots (e.g. Kardashev 1962; Biretta et al. 1991; Carilli et al. 1991). The spectral index found for this extended component by Körding et al. (2005) (from 8.4 to 15 $\mathrm{GHz}$ ) is $\alpha=0.42$ : slightly less steep, but broadly consistent. Looking at the e-MERLIN point source spectrum separately, at around $-2<\alpha<-2.5$, the spectral index is quite inverted, which could be due to Synchrotron Self Absorption (SSA) or free-free absorption. Indeed, had the core been resolved the source would have not been compact enough for SSA to be a viable mechanism to explain the absorption, as the magnetic fields required would have been orders of magnitude too high to explain the observed brightness temperature.

As it seems we have correctly identified the core we correlate this source position with multi-wavelength data.

\section{MULTI-WAVELENGTH OBSERVATIONS}

As M94 is relatively close, it is a well-studied source, observed by many different instruments. We are interested in M94* and thus attempt to match the $\left(0.15^{\prime \prime}\right)$ e-MERLIN res- 


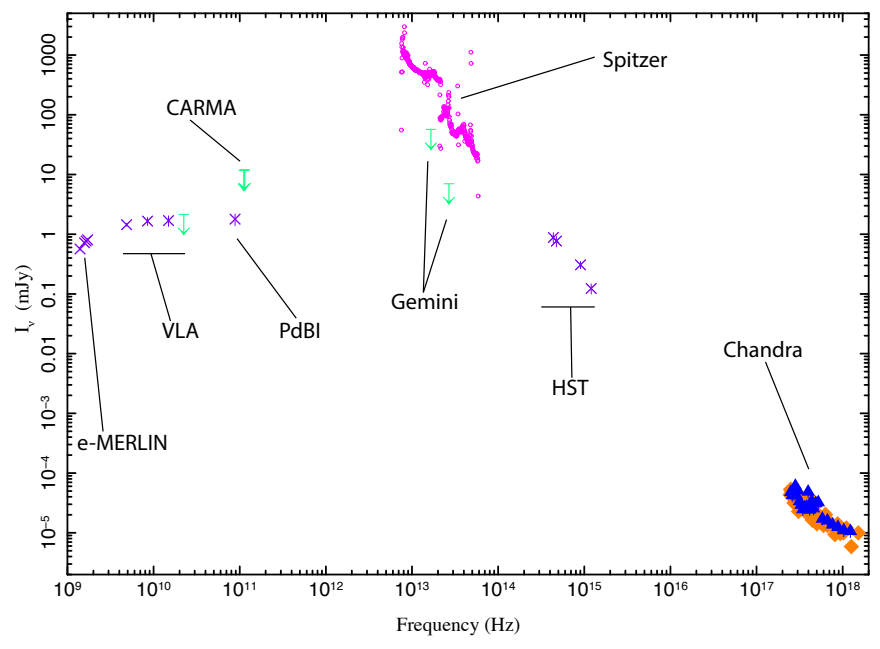

Figure 3. Multi-wavelength Spectral Energy Distribution (SED) of M94*, data only. For comparison we also include Spitzer data here (magenta open circles), which we will consequently ignore, as the two Gemini Michelle upper limits are clearly far better constraining. For details on all the data used, including precise observing frequencies, fluxes, expected resolutions, observation dates and an explanation of the upper limits, see Table 1 and its caption.

olution in the other wavebands in our selection of the available observations. The resulting SED (Figure 3) includes almost exclusively observations with high angular resolution (mostly sub-arcsecond, or $\lesssim 23$ pc at the distance to M94, down to $0.1^{\prime \prime}$, or $2.3 \mathrm{pc}$ in optical). The exceptions to this high resolution are the millimetre observations, that are the only measurements available for this source, but were obtained in compact configurations, i.e. originally optimised for different science goals. However as we will explain below we are confident the millimetre data accurately represents the M94* flux. Details of the observations are in the relevant subsections below, and are summarised in Table 1.

\subsection{Millimetre}

At millimetre wavelengths we have observations from the Plateau de Bure Interferometer $(\mathrm{PdBI})^{5}$ at $\sim 90 \mathrm{GHz}$ and the Combined Array for Research in Millimeter Astronomy (CARMA) at $\sim 110 \mathrm{GHz}$.

\subsubsection{PdBI}

The central region ( 130" diameter) of M94 was observed with 5 PdBI antennae in its D configuration during autumn 2013 (PI Van der Laan). Observations were centred at $88.8 \mathrm{GHz}$, with a $3.6 \mathrm{GHz}$ initial bandwidth at $2 \mathrm{MHz}$ resolution given the WIDEX correlator. Data reduction was done via the dedicated PdBI pipeline. The output was checked, and generally found to be excellent. Four out of six observational tracks included an absolute flux calibrator (MWC349 and/or LKHa101), and those tracks could be used to also

5 Now known as the NOrthern Extended Millimeter Array (NOEMA), after a recent upgrade to $>6$ antennae. flux calibrate the other two tracks. Overall the absolute flux error can be assumed to be less than $10 \%$. Finally, data quality limits were set to limit the influence of bad observations. This way $\sim 10-20 \%$ of visibilities were dismissed. The observing time remaining after calibration and reduction totals 13.3 hours spread over 11 pointings nearly uniformly, giving 1.2 hours for the central pointing.

A continuum uv-table was constructed from the remaining WIDEX visibilities, where $160 \mathrm{MHz}$-wide frequency ranges covering $\mathrm{HCN}(1-0)$ and $\mathrm{HCO}+(1-0)$ line emission (at $88.54 \mathrm{GHz}$ and $89.10 \mathrm{GHz}$, respectively) were excluded. This leaves a $3.3 \mathrm{GHz}$ total bandwidth. The sensitivity across the full $3.3 \mathrm{GHz}$ bandwidth is $0.065 \mathrm{mJy} /$ beam. Strong emission was found at position RA $12 \mathrm{~h} 50 \mathrm{~m} 53.068 \mathrm{~s}$, Dec $41 \mathrm{~d} 07 \mathrm{~m} 12.88 \mathrm{~s}$, near the phase centre of the observations. This emission was investigated with the GILDAS* routine uv_fit. The emission is unresolved (i.e. is a point-source) with a flux of $2.0 \mathrm{mJy}$. By measuring the flux in the uv-plane the fitting error remains small, and is only truly dominated by the absolute flux error.

Even though the resolution of the PdBI in D configuration is at least an order of magnitude lower than those of the measurements in the other wavebands (beam PSF is 5.7" by $4.6^{\prime \prime}$ ), no other significant continuum signal was found within the central $40^{\prime \prime}$ radius. In addition the positional accuracy of the flux measurement is an order of magnitude higher than the PSF size, since it was performed in the UVplane. Although this unresolved measurement may include some flux from the secondary source observed in radio, extrapolating the tightly correlated spectral index in Figure 2 this non-core source should contribute no more than $\sim 0.4$ mJy at these frequencies. Due to the error in absolute flux measurement the true flux density would lie between 1.8-2.2 $\mathrm{mJy}$, however the core only flux could in reality be $\sim 0.4 \mathrm{mJy}$ lower. Hence we will use a flux density of $1.8 \pm 0.4 \mathrm{mJy}$ for the PdBI measurement, so we can be sure this data point is a good representation of the core emission at this particular frequency, despite the larger PSF.

\subsubsection{CARMA}

The continuum observations at $111.5 \mathrm{GHz}$ and $114.5 \mathrm{GHz}$ presented in this paper have been obtained through the CArma and NObeyama Nearby galaxies (CANON) CO (10 ) Survey. In the CANON survey, data from the CARMA and Nobeyama Radio Observatory 45-meter (NRO45) single dish telescope are combined to image the centres of nearby spiral galaxies in the $(\mathrm{J}=1-0)$ transition of ${ }^{12} \mathrm{CO}$ (Koda et al. in prep). Due to its higher sensitivity on the relevant spatial scales, here we present only the CARMA data for the centre of M94.

The CANON observations of M94 at CARMA were taken in eight executions in the $\mathrm{C}$ and $\mathrm{D}$ configurations from January 2007 through March 2008 and are described in more detail in Donovan Meyer et al. (2013). The survey was designed to provide continuous velocity coverage around the CO (1-0) line with high spectral resolution and used wide continuum windows for calibration. Here we present measurements from the wide continuum windows in both the upper and lower sidebands. The windows are each $406 \mathrm{MHz}$ wide and are centred at $111.5 \mathrm{GHz}$ and $114.5 \mathrm{GHz}$. The field 


\begin{tabular}{|c|c|c|c|c|c|c|c|}
\hline Band & Instrument & Observed at & Flux (mJy) & Error (mJy) & Scale (") & Date & Reference \\
\hline \multirow[t]{8}{*}{ Radio } & e-MERLIN (L band) & $1.40 \mathrm{GHz}$ & 0.57 & 0.030 & 0.15 & $5 / 2014$ & $\mathrm{a}$ \\
\hline & e-MERLIN (L band) & $1.59 \mathrm{GHz}$ & 0.72 & 0.038 & 0.15 & $5 / 2014$ & $\mathrm{a}$ \\
\hline & e-MERLIN (L band) & $1.65 \mathrm{GHz}$ & 0.77 & 0.039 & 0.15 & $5 / 2014$ & $\mathrm{a}$ \\
\hline & e-MERLIN (L band) & $1.72 \mathrm{GHz}$ & 0.81 & 0.043 & 0.15 & $5 / 2014$ & $\mathrm{a}$ \\
\hline & VLA (C band) & $4.86 \mathrm{GHz}$ & 1.44 & 0.082 & 0.4 & $10 / 1983,1 / 1985$ & b \\
\hline & VLA (X band) & $8.49 \mathrm{GHz}$ & 1.65 & 0.26 & 0.24 & $6-10 / 2003$ & $\mathrm{c}$ \\
\hline & VLA (U band) & $14.9 \mathrm{GHz}$ & 1.70 & 0.34 & 0.15 & $1 / 2001$ & $c, d$ \\
\hline & VLA (K band) & $22.4 \mathrm{GHz}$ & $2.15^{\dagger}$ & $0.718^{*}$ & 0.96 & $2 / 2000$ & $\mathrm{~b}$ \\
\hline \multirow[t]{3}{*}{ Millimetre } & PdBI & $88.8 \mathrm{GHz}$ & 1.8 & 0.4 & $5.7 \times 4.6$ & $8-9 / 2013$ & e \\
\hline & CARMA & $111.5 \mathrm{GHz}$ & $11.1^{\dagger}$ & $3.7^{*}$ & $3.3 \times 2.3$ & $1 / 2007-3 / 2008$ & $\mathrm{a}$ \\
\hline & CARMA & $114.5 \mathrm{GHz}$ & $11.7^{\dagger}$ & $3.9^{*}$ & $3.2 \times 2.3$ & $1 / 2007-3 / 2008$ & $\mathrm{a}$ \\
\hline \multirow[t]{2}{*}{ Infrared } & Gemini Michelle (Q band) & $18.1 \mu \mathrm{m}$ & $57^{\dagger}$ & $(19)$ & 0.8 & $4 / 2007$ & $\mathrm{~g}$ \\
\hline & Gemini Michelle ( $\mathrm{N}$ band) & $11.2 \mu \mathrm{m}$ & $13^{\dagger}$ & (3) & 0.5 & $4 / 2007$ & g \\
\hline \multirow[t]{2}{*}{ Optical } & $H S T$ STIS & $6867 \AA$ & 0.881 & 0.176 & 0.1 & $6 / 2002$ & $\mathrm{~h}$ \\
\hline & $H S T$ STIS & $6295 \AA$ & 0.768 & 0.154 & 0.1 & $6 / 2002$ & $\mathrm{~h}$ \\
\hline \multirow{2}{*}{ Ultraviolet } & $H S T$ ACS F330W & $330 \mathrm{~nm}$ & 3.1 & 0.043 & 0.5 & $6 / 2003$ & $\mathrm{i}$ \\
\hline & $H S T$ ACS F250W & $250 \mathrm{~nm}$ & 0.123 & 0.024 & 0.5 & $6 / 2003$ & $\mathrm{i}$ \\
\hline X-ray & Chandra-ACIS & $1-8 \mathrm{keV}$ & see Fig 4 & & 1.35 & $5 / 2000,2 / 2009$ & $\mathrm{j}$ \\
\hline
\end{tabular}

Table 1. Summary of observations of the multi-wavelength data included in the nuclear SED in Fig 3. For completeness we quote in parentheses the Gemini Michelle $1 \sigma$ errors, however these are unused as we use the two data points as upper limits (see text). The other three upper limits used (VLA K band and CARMA) are $3 \sigma$ and the values quotes in the error column here denote the image RMS and are marked with an asterisk. The millimetre observations as well as the upper limits may include contributions from the extended source, described in section 2.2. References: a) This paper, b) NRAO VLA Archive Survey, c) Körding et al. (2005), d) Nagar et al. (2002, 2005), e) van der Laan et al. (2015), g) Asmus et al. (2014), h) Constantin \& Seth (2012), i) Maoz et al. (2005); Eracleous et al. (2010), j) Eracleous et al. (2002).

$\dagger$ Upper limit.

* Image RMS.

of view of the CARMA 19-pointing mosaic is $2.3^{\prime}$, centred on the position of M94.

The reduction was done using the usual CANON calibration routines in miriad which were adjusted to calibrate the continuum windows rather than the spectral line windows. The bandpass calibrators were 3C273 and J0927+390, with phase calibrators J1310+323 and J1159+292. The visibility flagging was not excessive.

The beam size in the $111.5 \mathrm{GHz}$ image is $3.3^{\prime \prime}$ by $2.3^{\prime \prime}$ and the beam size in the $114.5 \mathrm{GHz}$ image is $3.2^{\prime \prime}$ by $2.2^{\prime \prime}$. The images were created by inverting the visibilities with robust $=-2$ (a weighting that gives the less-well-populated longer baselines equal weighting and so pushes for a smaller beam, at the cost of slightly higher RMS). The RMS values measured in the centre of the maps are $3.7 \mathrm{mJy}$ at 111.5 $\mathrm{GHz}$ and $3.9 \mathrm{mJy}$ at $114.5 \mathrm{GHz}$. There are no continuum sources in either image within the CARMA field of view.

\subsection{Infrared}

Although Spitzer IRS spectral data is available, at a slit width of $3.6^{\prime \prime}$ the resolution of this instrument is relatively coarse and sure to be dominated by starlight and/or dust in the IR. Asmus et al. (2014) analysed archival data from the $8.1 \mathrm{~m}$ ground-based Gemini telescopes, as part of the Sasmirala (Subarcsecond mid-infrared view of local active galactic nuclei) survey. The survey features $N$ and $Q$ band M94 observations, obtained in 2007 with the Michelle spectrograph, offering nuclear/continuum flux measurements at 12 and 18 $\mu \mathrm{m}$. These flux measurements were obtained using the MIRРнот IDL routine that fits 2D Gaussians + a constant to the compact nuclear component in an attempt to subtract non- nuclear emission. Compared to the Spitzer measurements, the Sasmirala data constrains the M94* nuclear flux to a level 10 times lower. However Asmus et al. note that the central 100 pc of M94 is completely dominated by extended starlight and so, also considering the range of possible other origins of flux in this waveband (see Section 4), we will use these data points as upper limits.

It is worth noting that Richings et al. (2011) do not find evidence for a point source in the near-IR (in the Hubble F160W filter, at around $1.6 \mu \mathrm{m})$.

\subsection{Optical}

The two optical measurements included represent a spectrum obtained with the HST Space Telescope Imaging Spectrograph (STIS). The data were reduced and reported by Constantin \& Seth (2012) being sampled over a rectangular aperture of $0.25^{\prime \prime}$ by $0.1^{\prime \prime}$. Considering all the possible origins of "pollution" of the optical (and UV) by non-jet sources we do not fit the detailed spectrum reported in that work, but use the spectrum end-points only, assuming $20 \%$ errors, to get an indication of the normalisation at these energies. In any case we do not expect the jet to be dominant in the optical/UV and for these reasons we do not fit the optical/UV data with a jet, but with an accretion disk, in order to take care of this normalisation (see Section 4).

\subsection{Ultraviolet}

In UV we use data reported in Eracleous et al. (2010) that includes data from the HST Advanced Camera for Surveys (ACS), which has been automatically reduced by the Space 


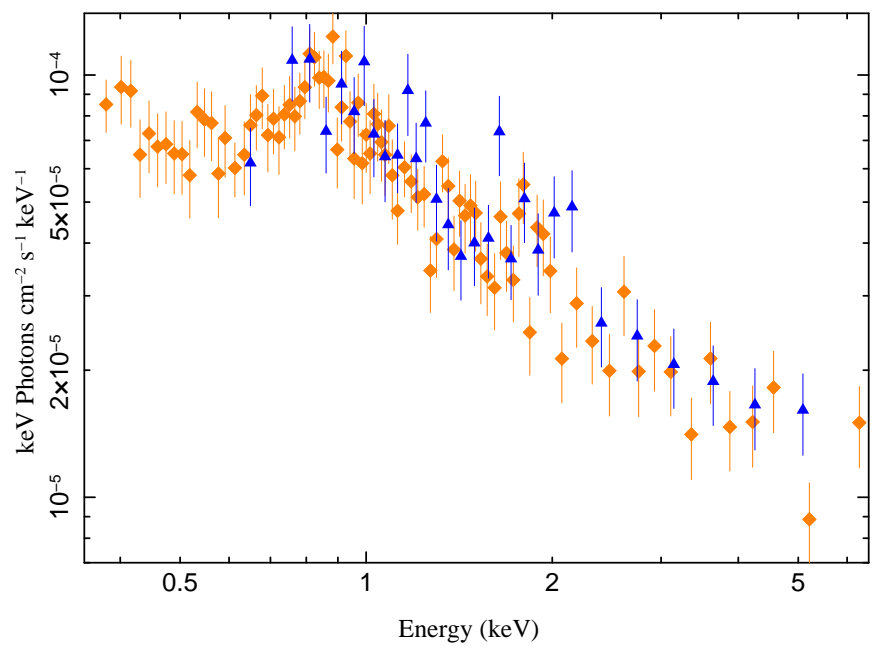

Figure 4. Background-subtracted X-ray SED of M94*, covering the full useable $0.36-8 \mathrm{keV}$ Chandra range, binned to a minimum $\mathrm{S} / \mathrm{N}$ of 4.5. The data was unfolded using the same model as used in Figures $5-7$. We show the full X-ray range here for clarity, but only use the $>1 \mathrm{keV}$ data in this work (see text). Orange diamonds are Eracleous observations from 2000 while blue triangles are Jenkins observations from 2008.

Telescope Science Institute (STScI) pipeline as well as aperture photometry that has been done by Maoz et al. (2005), within a 10 pixel $\left(0.27^{\prime \prime}\right)$ radius centred on the unresolved point source. These UV data are also used for SED fitting in Nemmen et al. (2014). Eracleous et al. (2010) employed two different methods to de-redden the data, a starburst extinction law (Calzetti et al. 1994) and a maximum correction corresponding to the Milky Way law of Seaton (1979). For our fluxes we take the average of the latter and the undereddened flux and use these extremes to determine the error bar sizes.

\subsection{X-ray}

Using the Advanced CCD Imaging Spectrometer (ACIS), Chandra has observed M94 three times since its launch: twice in 2000 (ObsID 808, $47.37 \mathrm{ks}$ and ObsID 410, 2.31 $\mathrm{ks}$ ) and once in 2008 (ObsID 9553, $24.35 \mathrm{ks}$ ). Considering the relatively short exposure for ObsID 410 we choose to ignore this observation and reduce the other two using the standard CIAO (Chandra Interactive Analysis of Observations) software, v4.8 (Fruscione et al. 2006) and the CALDB version 4.7.2 calibration files. After extracting the spectra from the individual observations (with the CIAO tool SPECEXTRACT) we fit for both datasets simultaneously, however the 2000/2008 observations have been performed in VFAINT/FAINT mode and ACIS-S/ACIS-I respectively.

For both datasets we have to choose an appropriate extraction region. The normal pixel size of the CCD is $0.49^{\prime \prime}$, while the PSF should be slightly smaller. We are interested in a point source, however the error in the absolute photometry is of the same order as the pixel size. In addition there is an energy dependence of the Chandra PSF such that the hard end of the PSF may have large wings that will fall beyond the extraction region if the latter is chosen too small. However the field is crowded, with close neighbour- ing sources limiting the size of the extraction region. Hence, we choose a circle that maximises surface area and includes the coordinates of the nucleus (at $15 \mathrm{GHz}$, which closely align with the PdBI observations, see above) but whose radius does not exceed the half-way points between the nucleus and the two closest neighbouring sources. The resulting extraction region is centred on coordinates 12:50:53.066 $+41: 07: 12.73$, has a radius of $1.35^{\prime \prime}$ (see Fig. 1). To estimate the background we use 5 circular regions of $2.75^{\prime \prime}$ radius, randomly placed at empty regions roughly $1^{\prime}$ away from the target. The final X-ray spectra are presented in Figure 4.

The usable energy range of Chandra is $0.36-8 \mathrm{keV}$. Below this range electronic noise dominates the signal from the sky X-rays, while above $8 \mathrm{keV}$ there is negligible instrument response (Doane et al. 2004). In Figure 4 we see two components below $1 \mathrm{keV}$ (that can be modelled well as a thermal plasma, at $\sim 0.1$ and $\sim 0.6 \mathrm{keV}$, see Roberts et al. 1999; Pellegrini et al. 2002) contaminating the spectrum. These excesses observed in the X-ray SED seem due to larger-scale emission $^{6}$, e.g. hot, collisionally excited ISM, iron L and/or Neon lines (at $\lesssim 1 \mathrm{keV}$ ) and oxygen lines at lower temperatures, likely to be due to star formation and perhaps only indirectly related to the AGN by shocks. For an AGN at such low accretion rates there should be no soft X-ray contribution from the accretion disk blackbody emission, so these lower energies can be safely ignored by our model. Therefore we truncate the spectrum to consider only X-rays $>1$ $\mathrm{keV}$.

\subsection{Variability}

The data in our SED are non-simultaneous. As explained in the introduction, this need not be a problem, since AGN tend to go through their evolution on extremely long timescales. LINERs can however be highly variable on timescales of months to years (with typical peak-to-peak amplitudes from a few to 50\%), particularly in the UV and Xray bands (e.g. Maoz et al. 2005; Pian et al. 2010; Younes et al. 2011). Therefore we will briefly discuss the variability in the different domains, starting at low energy.

In the the radio through millimetre we may observe small fluctuations in the jet, consistent with adiabatic expansion or from normal physical variations in the input power, jet structure or accretion rate, travelling away from the nucleus as "wiggles" in the spectrum. Markoff et al. (2008) found evidence for such "waves of variability" in M81*. However we see a consistently inverted spectrum, that increases almost perfectly monotonically with energy, at least up to PdBI frequencies. Hence, it seems unlikely that the variability in this domain should be significant at the time scales we are looking at, even though the observations used are spread over the past three decades in time (perhaps indicating a remarkably steady jet).

In infrared and optical bands we do not expect to see much variation, since we will mainly see galaxy light here. In the UV however, variation is expected for AGN. For M94*, Maoz et al. (2005) measured a variability of $5 \%$ and $1 \%$ in the HST F250W and F330W ACS bands respectively, confirming an AGN could be responsible for a significant contri-

${ }^{6}$ Unrelated to the extended radio emission in Section 2.2. 
bution in the UV bands. In addition, between 1993 and 2003 these authors observe long term variability of a factor of 2.5 in the UV, also indicative of an AGN. We do not expect these variations to be due to the jet. Moreover, for our modelling, variability in the IR-to-UV can be mostly ignored: As the jet is not expected to be the dominant component here (see below for discussion) it will not tell us much about the physics we want to study in this work. As will be explained in Section 4 we model these energies with a thin accretion disc as the dominant component and so the jet model results will not be sensitive to variability in the UV.

The X-ray variability of M94* has been examined several times before using different instruments. Roberts et al. (1999) found no evidence for long-term (multi-year) variability comparing ROSAT and ASCA data, constraining the amplitude of the variation of observations four years apart to $\lesssim 30 \%$ ). Also Pellegrini et al. (2002) found no evidence of 2-10 keV flux variations between $A S C A$ and BeppoSAX observations, separated by five years. These instruments may conceal variation, however, due to a larger ratio of diffuseto-core emission expected to be included in their relatively coarse PSFs, compared to Chandra and XMM-Newton. Our Chandra observations were obtained 8 years apart. However integrating and comparing the 1-6 keV X-ray flux of these individual observations, we only see evidence for $\sim 2 \%$ long term variability. Eracleous et al. (2002) also look into the short-term variability for their dataset and constrained the excess variance to $0.06 \pm 0.04 \mathrm{~s}^{-1}$, i.e at the $5-10 \%$ level only at timescales of a few hours.

From the above considerations we believe the variability in M94* is insufficient to significantly influence our conclusions, as evidence suggests that it is a remarkably stable source. Hence the results from fitting the SED (Section 5) should be robust enough to interpret and compare with other sources studied with MNW05.

\section{JET PARADIGM}

For spectral fitting we use the Interactive Spectral Interpretation System ISIS (Houck \& Denicola 2000), compiled with XSPEC (Arnaud 1996) 12.8.2 libraries. ISIS allows us to forward-fold the model flux predicted by MNW05 through the Chandra detector response matrices during fitting (while all non-Chandra data is fitted directly).

For details of the MNW05 model we refer to previous works of e.g. Markoff et al. (2005); Maitra et al. (2009a); van Oers et al. (2010), but we will briefly describe the main parameters here.

There are several physical parameters to the model that are fixed by observations, such as the $\mathrm{BH}$ mass $\left(M_{\mathrm{BH}}\right)$, the distance $d$ and jet inclination $i$. The jet itself is similar to a Blandford \& Konigl (1979) scenario, compact and stratified, consisting of multiple self-absorbed zones and has a length $z_{\max }$. All length scales are in units of gravitational radii $\left(\mathrm{r}_{g}=G M_{\mathrm{BH}} / c^{2}\right)$. The base of the jet, or jet "nozzle", is cylindrical, with radius $\mathrm{r}_{0}$ and height $h_{0} / r_{0}$. The power entering the jets is represented by $\mathrm{N}_{\mathrm{j}}$, which is the dominant fitted parameter. $\mathrm{N}_{\mathrm{j}}$ scales with the accretion power. The energy fed into the nozzle is divided between the kinetic and internal pressures, assuming the electrons are mainly responsible for the radiation, while cold (non-relativistic) protons carry the bulk kinetic energy. The ratio of the magnetic and particle energy densities is referred to as the equipartition factor $k=U_{B} / U_{e}$, with $U_{B}=B^{2} / 8 \pi$ the magnetic energy density, $B$ the magnetic field, and $U_{e}$ the electron energy density. The nozzle electrons are assumed to be in a quasi-thermal, mildly relativistic Maxwell-Jüttner velocity distribution of temperature $T_{\mathrm{e}}$, but at some length $z_{\text {acc }}$ from the jet-base we assume $60 \%$ of the electrons are accelerated into a powerlaw tail $\left(N(E) \propto E^{-p}\right.$, where $p$ is a free parameter $)$, assuming a process like diffusive shock acceleration (e.g. Jokipii 1987). However we remain agnostic about the true acceleration process and parametrise the acceleration efficiency with a factor $\epsilon_{\text {scat }}$ that we fit for and in doing so absorb the unknowns. In all segments beyond the nozzle the jet is assumed to expand quasi-isothermally (see Crumley et al., in prep.), causing a longitudinal pressure gradient that accelerates the bulk-plasma asymptotically to Lorentz factors of 2-3 (derived from the Euler equations; Falcke 1996).

The MNW05 framework incorporates cooling due to expansion, and synchrotron and IC losses under the assumption of a constant injection of fresh, high energy power-law leptons in each segment after the acceleration front. However if the cooling is fast, the injection rate may not be high enough to compensate for the cooling. If the dynamical timescale $t_{\mathrm{dyn}}$ of a segment (i.e. the lepton residence time) is at least of the order of the lepton synchrotron lifetime $t_{\mathrm{syn}}$, synchrotron losses will be significant. As a consequence the index of a steady state power-law electron distribution will be steepened by unity, from $p$ to $p+1$. The turnover point where the indices steepen is referred to as a cooling break and should occur around $E_{\mathrm{br}}=\frac{24 \pi m^{2} c^{3}}{\sigma_{T} B^{2} t_{\mathrm{dyn}}}$ (Kardashev 1962).

We also multiply the outflow model by an absorption model (phabs in ISIS), parametrised by the line-of-sighthydrogen density $N_{\mathrm{H}}$ (see Sec. 4.1 for details), which shows the biggest contribution in the softest X-rays.

We also include an optically thick, geometrically thin multi-colour accretion disk with a temperature profile of $T \propto$ $R^{-3 / 4}$, following Shakura \& Sunyaev (1973); Mitsuda et al. (1984); Makishima et al. (1986). This disk is parametrised by an inner radius $\mathrm{r}_{\text {in }}$ (with a temperature $\mathrm{T}_{\text {in }}$ ) and an outer radius $\mathrm{r}_{\text {out }}$. Although the photons coming from this disk are available for Compton up-scattering by the leptons in the base of the jet, for M94* the contribution of this effect to the total model flux is insignificant. Furthermore, for LINERs the canonical "big blue bump" (e.g. Elvis et al. 1994) is often missing (e.g. Ho 1999; Eracleous et al. 2010), hinting at a truncation/disappearance of a thin accretion disk. For LINERs the UV spectrum in $\log v-\log \left(v F_{v}\right)$ representation is usually of comparable amplitude to the X-ray spectrum (see e.g. Fernández-Ontiveros et al. 2012), however M94* is $~ 100$ times brighter in UV than in X-ray. This is understandable as for most galaxies the flux in the IR-to-UV bands is expected to be an amalgamation of both galactic (e.g. stellar population and dust) and nuclear contributions (e.g. jet synchrotron, thermal radiation from a minor accretion disk, as well as reprocessed radiation from dust in a torus or narrow line region). In theory it should be possible to use a combination of stellar population synthesis templates (e.g. Bruzual \& Charlot 2003) and other models to account for all these components accurately. However the available data is insufficient to accurately disentangle the ex- 


\begin{tabular}{lccc}
\hline Parameter & Value & Unit & Reference \\
\hline$d$ & 4.8 & $\mathrm{Mpc}$ & $\mathrm{a}$ \\
$M_{\mathrm{BH}}$ & 6.77 & $10^{6} \boldsymbol{M}_{\odot}$ & $\mathrm{b}$ \\
$i$ & 29 & ${ }^{\circ}$ & $\mathrm{c}$ \\
$N_{\mathrm{H}}$ & $1.41-3.3 \times 10^{20}$ & $\mathrm{~cm}^{-2}$ & $\mathrm{~d}$ \\
\hline
\end{tabular}

Table 2. Summary of observable parameter values, and references: a) Eracleous et al. (2010); b) Kormendy \& Ho (2013); c) Erwin (2004); Trujillo et al. (2009); d) Stark et al. (1992); Eracleous et al. (2002).

act flux contributions in this energy range. Hence we make use of the (quasi-)thermal thin disk to model these 2 Gemini upper limits and $4 H S T$ data points and account for the normalisation at this energy. In effect this disk subsumes all unseparated non-jet contributions from all the components mentioned above (stars, dust, etc). This approach allows us to focus on the broadband spectrum and implied physics of the compact jet source only.

In the X-ray band, to account for the different Chandra modes/instruments/varying PSFs as well as possible source variability we allow a constant to determine the relative normalisation for the 2008 data compared to the 2000 dataset, as the latter has significantly more counts due to the longer observing time. Fitting data of different epochs should have the added benefit of further averaging out some of the expected variability in the X-rays (which is small to begin with, see Sec. 3.6).

\subsection{Fixed and limited input parameters}

As mentioned above we fix the observable model parameters, or put limits on the ranges allowed using results from the literature. These values and ranges are listed in Table 2 .

Tonry et al. (2001) determined the distance $d$ to M94 from surface brightness fluctuations. We however use a corrected value reported in Eracleous et al. (2010), who subtracted $0.16 \mathrm{mag}$ from the distance modulus in Tonry et al., following Jensen et al. (2003).

The black hole mass $M_{\mathrm{BH}}$ is a dynamical measurement quoted from Kormendy \& Ho (2013).

The compact jets in M94 have not been resolved and therefore the jet inclination is unknown. Moreover there is no correlation expected between the AGN jet inclination and the galaxy disk inclination (Kinney et al. 2000; Netzer 2015). The jet inclination could however be causally linked to the inclination of the inner galaxy disk. Based on the shape of the inner ovally distorted disk Erwin (2004) reported a galaxy inclination of $i=35^{\circ}$. Consistent with this value, de Blok et al. (2008) found the galaxy disk inclination decreasing from $\sim 50-29^{\circ}$ from the outer disk to the inner disk, based on H I measurements. In our fits, lower inclinations are preferred to accommodate the relative flatness of the radio spectrum (higher inclinations yield a more inverted spectrum due to mild relativistic beaming). Hence for fitting we will use the bottom/inner disk value from de Blok et al. (2008) of $i=29^{\circ}$.

We limit the range for the absorption column $N_{\mathrm{H}}$, requiring it to fall between the value corresponding to Galactic absorption alone (Stark et al. 1992) and the upper limit that was found by (Eracleous et al. 2002), who fitted the
2002 Chandra spectrum data (that is also included in our SED) with an absorbed power-law.

With regards to the electrons we make a few assumptions: We use a particle distribution index $2<p<2.5$, consistent with diffusive shock acceleration (e.g. Heavens \& Drury 1988; Lemoine \& Pelletier 2003; Nemmen et al. 2014 and references there-in). Also, as we see no evidence in the SED of a high-energy, exponential cutoff, we will assume the acceleration of the leptons occurs efficiently, and set $\epsilon_{\text {scat }}=0.01$ in synchrotron dominated fits. This value is high enough to set the cutoff beyond the energy range covered by our M94* data. Lastly this model is only valid for electron temperatures $\mathrm{T}_{\mathrm{e}}$ greater than $m_{e} c^{2} / k_{\mathrm{B}}=5.94 \times 10^{9}$ $\mathrm{K}$, as below this value the electrons would mainly emit cyclotron radiation instead of synchrotron radiation and we do not model the former separately.

\section{RESULTS}

We use the ISIS script conf_loop to iteratively search for confidence intervals on free parameters, using a tolerance of $\Delta \chi=10^{-3}$. As our data were not obtained simultaneously, it is impossible to assess errors resulting from systematics due to possible variability in M94*. However, as explained in Section 3.6, we see evidence of only limited variability in this source. Hence we include all the $90 \%$ confidence limits obtained $^{7}$, in Table 3. The associated fits are displayed in Fig. $5-7$.

For most LLAGN it is difficult to constrain the dominant radiative mechanism (e.g. Plotkin et al. 2012), due to degeneracies within the possible radiative mechanisms. Firstly, within synchrotron there is an intrinsic degeneracy, where energy distributed into either the particle energy density or the magnetic energy density can work to enhance the spectral flux, since both an increase in lepton density (number of particles radiating), as well as a stronger magnetic confinement of these particles (individual particles radiating more) can increase the net flux. Secondly, both synchrotron and inverse-Compton processes can create power-law radiation. Hence, unsurprisingly, we find three statistically good models with acceptable physical model parameters. In the first fit (from here on referred to as SYN) the X-ray spectrum is well-approximated by optically thin synchrotron photons originating from the acceleration region at $z_{\mathrm{acc}} \sim 100 \mathrm{r}_{\mathrm{g}}$. The second fit has incrementally better statistics compared to the other two, and since the most salient difference is a significant synchrotron self-Compton (SSC) contribution in the softer X-rays we will refer refer to this fit as SYNCOM. Thirdly we obtained a fit where the X-rays are fully dominated by SSC (hereafter: COM). In the following we proceed to describe and compare these fits and explain why the SYN fit seems the most physically tenable. Consequently we will compare our M94* modelling with other sources previously modelled with MNW05 in Section 6.1.

7 We do not quote confidence limits on the acceleration zone distance $\left(z_{\text {acc }}\right)$ due to the segmented nature of the MNW05 jet. As the jet is composed of discrete zones, neither the location nor the confidence limits can be accurately determined. 

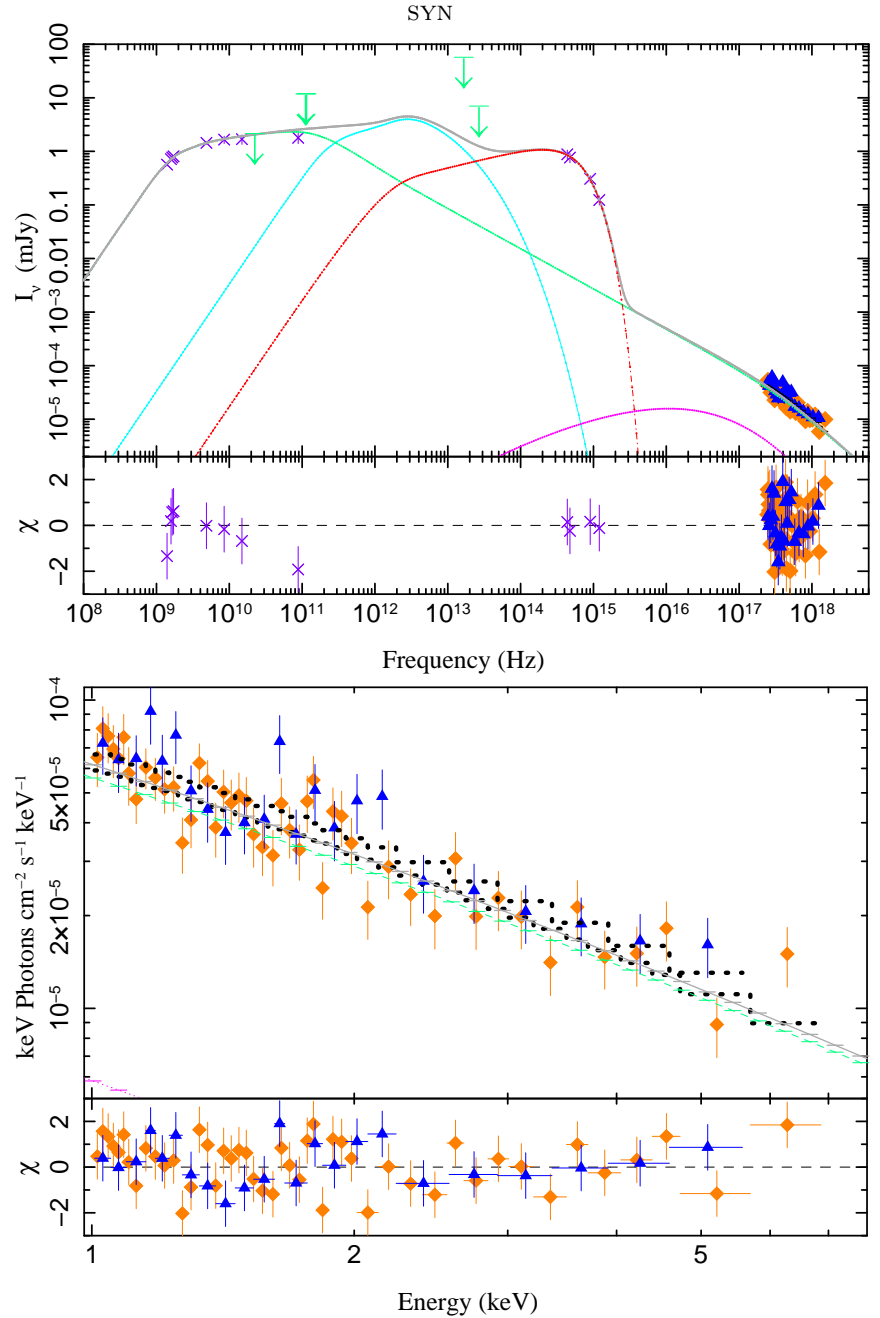

Figure 5. M94* multi-wavelength broadband (top) and X-ray only (bottom) SED and best-fit model for the SYN fit (see Table 3). The broadband SED is as in Figure 3 (without the Spitzer data). The bottom plot is a simple magnification of the upper plot, but with the energy scale in $\mathrm{keV}$ instead of frequency. Hence all lines, colours and symbols are the same in both plots and will be defined below. Individual components from the MNW05 model are shown with the following lines/colours: The cyan dotted curve is the jet pre-shock synchrotron contribution. The green solid curve is the post-shock synchrotron. The red dash-dotted curve represents all the contributions in the IR-to-UV, modelled by a multi-colour accretion disc. The magenta solid curve represents the Compton-up-scattered seed photons from both the synchrotron self-Comptonisation (SSC) of the pre-shock synchrotron and the accretion disc, although, considering the relative normalisations of those components, the latter only contributes weakly in these models. The solid grey line indicates the total of the above components (however not forward-folded through the detector response matrices). Only visible in the bottom plot, the black dashed lines show the forward-folded model fluxes (albeit only where data is present) for both X-ray data sets that are denoted by orange diamonds and blue triangles respectively (as explained in Fig 4).

\begin{tabular}{|c|c|c|c|c|}
\hline \multicolumn{5}{|c|}{ M94* } \\
\hline & Parameter (Unit) & SYN & SYNCOM & $\mathrm{COM}$ \\
\hline \multirow{8}{*}{ 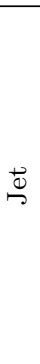 } & $\mathrm{N}_{\mathrm{j}}\left(10^{-5} L_{\mathrm{Edd}}\right)$ & $6.8_{-5.0}^{+0.8}$ & $28_{-14}^{+51}$ & $35_{-18}^{+9}$ \\
\hline & $\mathrm{T}_{\mathrm{e}}\left(10^{11} \mathrm{~K}\right)$ & $1.4_{-0.2}^{+0.3}$ & $1.0_{-0.1}^{+0.1}$ & $1.3_{-0.1}^{+0.2}$ \\
\hline & $\mathrm{r}_{0}\left(r_{\mathrm{g}}\right)$ & $3.7_{-1.7}^{+3.2}$ & $6.4_{-3.0}^{+3.1}$ & $1.0_{>-0.0}^{+1.1}$ \\
\hline & $k$ & $0.9_{-0.3}^{+10.9}$ & $0.10_{-0.05}^{+0.00}$ & $0.03_{-0.02}^{+0.11}$ \\
\hline & $\epsilon_{\text {scat }}$ & $0.01 *$ & $0.01^{*}$ & $8 \times 10^{-7}$ \\
\hline & $p$ & $2.49_{-0.12}^{>+0.01}$ & $2.44_{-0.03}^{+0.00}$ & $2.50_{-0.28}^{>+0.00}$ \\
\hline & $z_{\text {acc }}\left(r_{\mathrm{g}}\right)$ & 94 & 768 & 425 \\
\hline & $h_{0} / r_{0}$ & $1.5^{*}$ & $12_{-4}^{+20}$ & $0.7_{-0.1}^{+2.8}$ \\
\hline \multirow{3}{*}{$\frac{\frac{y}{y}}{\tilde{n}}$} & $\mathrm{r}_{\text {in }}\left(r_{\mathrm{g}}\right)$ & $37_{-8}^{+8}$ & $37_{-9}^{+9}$ & $36_{-10}^{+3}$ \\
\hline & $\mathrm{T}_{\text {in }}\left(10^{3} \mathrm{~K}\right)$ & $10.6_{-1.2}^{+1.0}$ & $10.6_{-0.9}^{+1.1}$ & $10.6_{-1.0}^{+1.5}$ \\
\hline & $\mathrm{r}_{\text {out }}\left(r_{\mathrm{g}}\right)$ & $10^{4 *}$ & $10^{4 *}$ & $10^{4 *}$ \\
\hline & & $78.4 / 73$ & $68.7 / 72$ & $81.2 / 72$ \\
\hline & & $(\simeq 1.07)$ & $(\simeq 0.95)$ & $(\simeq 1.13)$ \\
\hline
\end{tabular}

Table 3. Best-fit model parameters for M94* (see Section 4 for detailed description; The jet-related paramaters are: $\mathrm{N}_{\mathrm{j}}=$ the scaled jet power, $T_{e}$ is the electron temperature in the jet base, $\mathrm{r}_{0}$ is the radius of the jet base, $k$ is the ratio of thermal electron energy density to magnetic energy density, $\boldsymbol{\epsilon}_{\text {scat }}$ relates to acceleration efficiency, $p$ is the accelerated particle distribution index, $z_{\text {acc }}$ is the location where particle acceleration starts in the jets, $h_{0} / r_{0}$ is the scaled height of the jet base). An asterisk $\left(^{*}\right)$ indicates that a parameter was frozen at the value given. We failed to resolve both upper and lower limits of the error bars for parameters listed in italics, while we failed to resolve either an upper, or a lower bound for parameters with a greater-than sign (>, meaning the true confidence limit is farther removed from the parameter value than indicated by the limit value). To fit the e-MERLIN slope well at the lowest radio frequencies the length of the jet $z_{\max }$ was fixed to $10^{16.15} \mathrm{~cm}$ (to avoid over-interpreting the model we do not allow this parameter freedom to vary, see text).

SYN

The X-rays of the SYN fit are dominated by optically thin cooled synchrotron radiation, displaying a steepening of slope in the soft X-rays. Observing this break is consistent with findings in Russell et al. (2013) who predict such a break between the UV and the X-rays at the X-ray luminosities (in Eddington) that M94* portrays. The fit shows all confidence limits resolved, except for the upper limit of the particle distribution index $p$ (within the range allowed). A marginal SSC contribution is seen in the UV - soft Xrays, however this component is not needed to adequately fit the data and hence the jet height-to-base ratio $h_{0} / r_{0}$ was fixed to 1.5, consistent with previous works. At $k \sim 0.9$ the jet would be slightly sub-equipartition, however within the errors a magnetically dominated jet cannot be ruled out. Considering the large upper limit on the jet base radius, the nozzle appears less compact than in the other two jets.

\section{SYNCOM}

In absolute terms the SYNCOM model shows the best statistics of all three fits. The improvement in $\chi_{\text {red }}^{2}$ is mainly due to a lower residual for the submm PdBI data point due to a slightly flatter model radio spectrum. In the MNW05 model, mainly the inclination determines how flat or inverted the radio spectrum is, with higher inclinations leading to more 

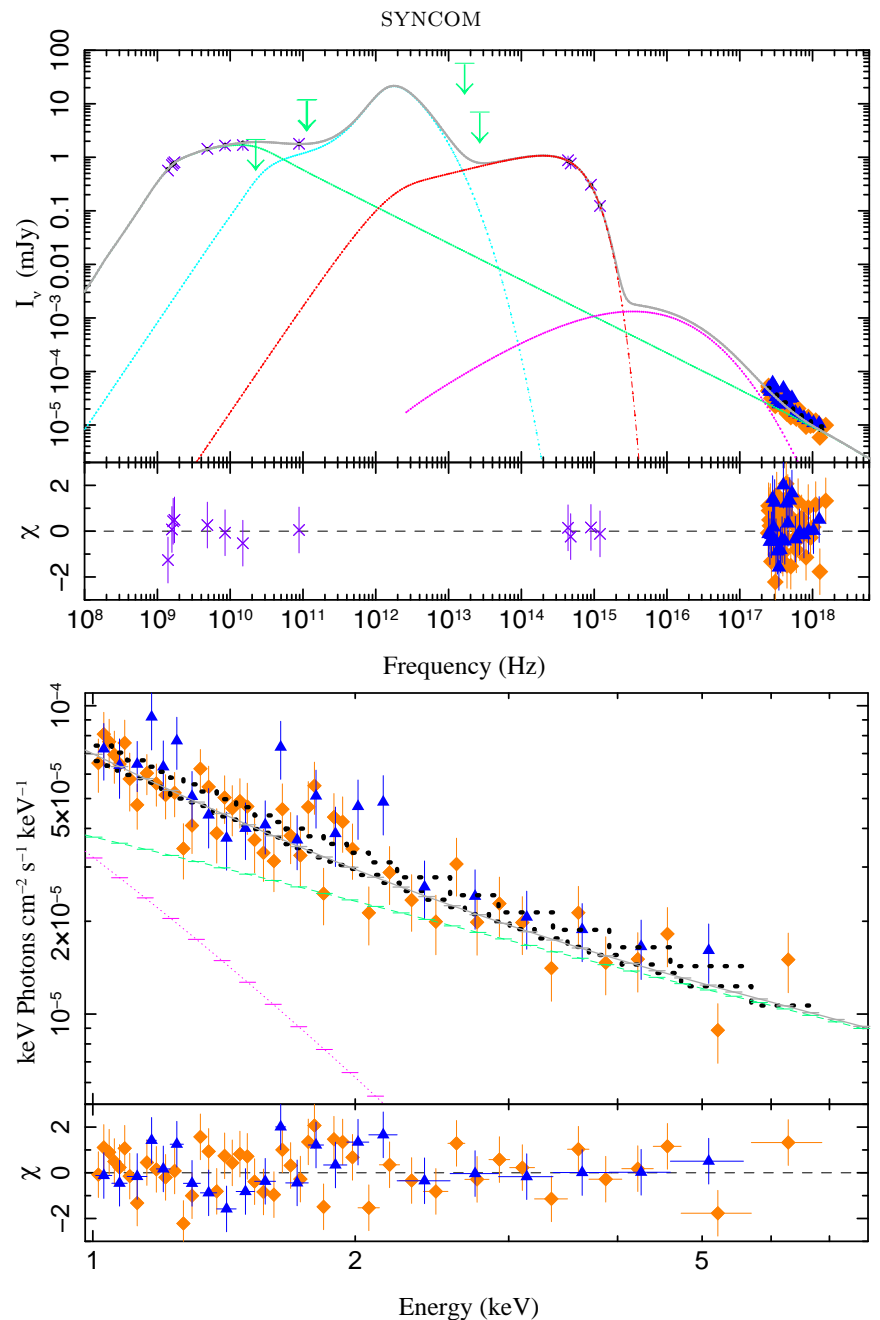

Figure 6. Multi-wavelength broadband (top) and X-ray only (bottom) SED and best-fit model of M94* for the SYNCOM model (see Table 3). Definitions of lines, colours and symbols are explained in Figure 5. Please note that in the top panel the (insignificant) SSC component has not been plotted below $\sim 2 \times 10^{12}$ $\mathrm{Hz}$, due to numerical breakdown of the model.

inverted radio spectra. However the transition from an optically thick to an optically thin dominated spectrum can locally flatten the radio spectrum somewhat at an energy just above this transition (compare the top panels of Figure 6 with those of Figure 5 and 7 ; in contrast to the latter two Figures, the grey line that denotes the total model flux is not inverted above $\sim 10^{11} \mathrm{~Hz}$ in Figure 6). In the SYNCOM fit, this transition occurs at an energy just below the PdBI observing frequency, tailoring the radio/submm model spectrum almost exactly to the data. The X-rays, on the other hand, do not show improved residuals at all. We consider the transition effect to be a model artefact and the consequent improved statistics are thus not due to the model better describing the underlying physical picture. Therefore we find it likely that this fit represents a local or "false" minimum. In addition, compared to the other two fits an extra SSC component is needed to fit the X-rays equally well. Using an extra fitting-component while not improving the actual model should generally be avoided to minimise the available
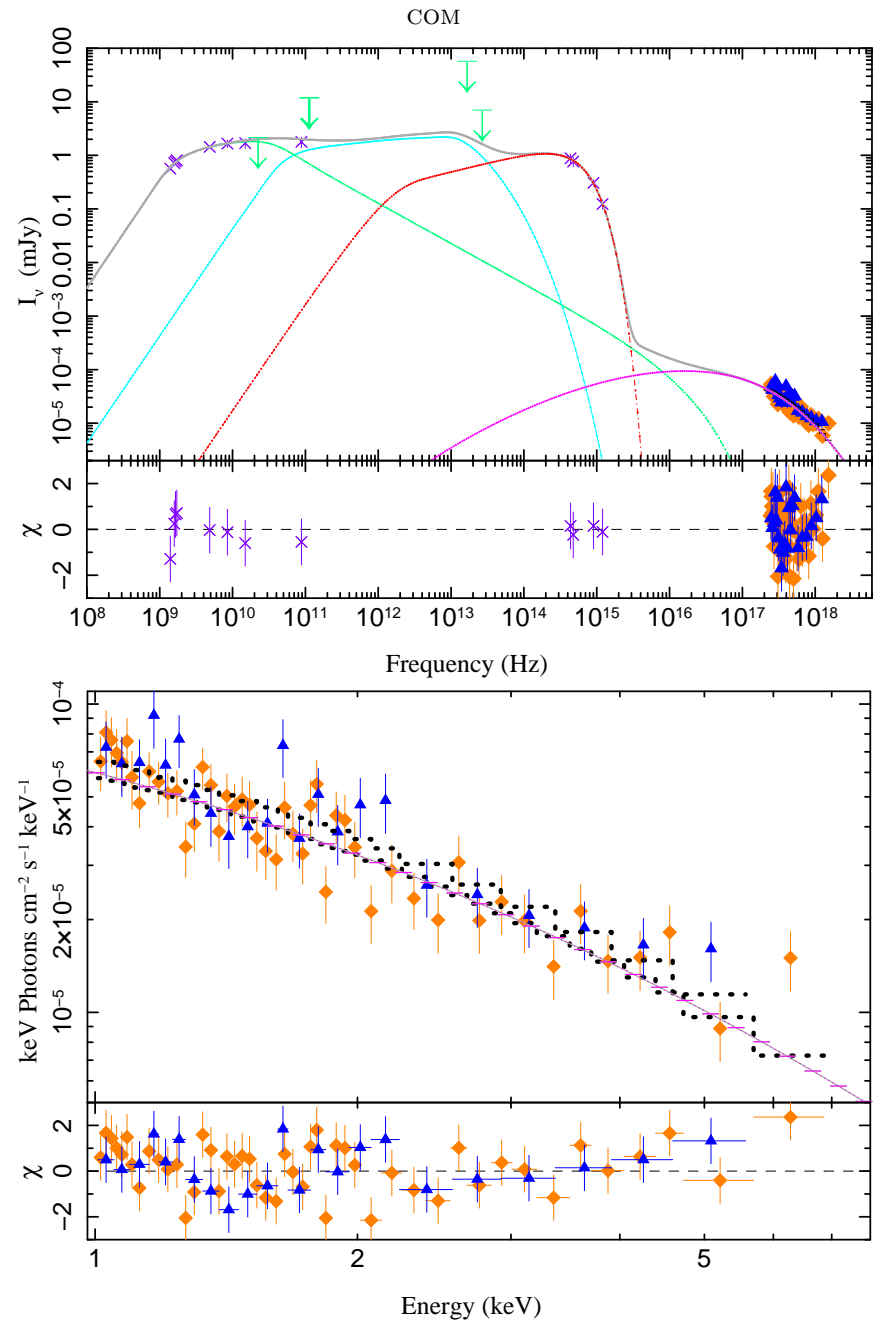

Figure 7. Multi-wavelength broadband (top) and X-ray only (bottom) SED and best-fit model of M94* for the COM model (see Table 3). Definitions of lines, colours and symbols are explained in Figure 5.

degrees of freedom and avoid degeneracy. Hence we will exclude the SYNCOM fit from detailed further analysis, but have included it here for completeness.

$\mathrm{COM}$

As is also clear from the SYNCOM fit above, a significant SSC contribution requires an increasingly compact nozzle. In the COM fit this is evident from the relatively small jet base radius that pushes the lower allowed value of $r_{0}=1$ $r_{\mathrm{g}}$. As this value is within the $6 r_{\mathrm{g}}$ innermost stable orbit of a Schwarzschild BH it suggests $\mathrm{M} 94^{*}$ is rapidly rotating. An even smaller value of $r_{0}$ would place the entire nozzle within the innermost stable orbit of even a maximally spinning "Kerr" black hole. The low value of the ratio of magnetic to particle distribution energies $k$ may however pose a bigger problem. Although values below unity could be possible, it is currently not quite clear what it means in the context of an MNW05 jet, that is assumed to be maximally dominated. Due to uncertainties regarding energy redistribution 
in the jet nozzle it is difficult to quantify whether a subequipartition flow is physical or perhaps an artefact due to simplifications in the model (also see discussion in Plotkin et al. 2015). In general, particularly for BHBs, the results are more consistent with equipartition. Thirdly, the acceleration efficiency parameter $\epsilon_{\text {scat }}$ needed to be fixed (within a truncated range $10^{-6}-10^{-8}$ ) to avoid the fit evolving towards a dominant optically thin synchrotron contribution in the X-rays (yielding a result similar to one of the two above). Consequently the optically thin synchrotron is not at all constrained by the X-rays. For the same reason the particle distribution index $p$ is not well-constrained. Although this fit seems to be pushing extremes, especially in terms of $r_{0}$ and $k$, we assert that within the uncertainties of the model this fit can not be excluded from consideration, particularly as this type of solution has been known to describe well other low luminosity sources like quiescent BHBs (Plotkin et al. 2015) and low luminosity AGN.

\section{Further remarks on fits}

As explained in Section 4 we assume the presence of an accretion disk and use its quasi-thermal flux to account for the IR-to-UV flux measurements, although additional physical components are expected to contribute. The obtained accurate fit of the optical and UV HST data suggests a thermal origin (perhaps the high energy cutoff of a stellar population), but considering the uncertainties we draw no further conclusions from the obtained disk parameters ${ }^{8}$. We only list them in Table 3 for completeness.

As touched on above: Our fits to the radio spectrum suggest that the inclination chosen $\left(29^{\circ}\right)$ could be on the high side. A lower inclination would yield a flatter radio spectrum and hence better accommodate the PdBI data point. We have set the inclination using observations from de Blok et al. (2008), who quote a value specific for the inner disk to which we assumed the jet may be causally linked (emphasising again that the jet and galaxy disk inclination are not expected to be correlated). For completeness we note that Trujillo et al. (2009) quotes a lower inclination of $8^{\circ}$, inferred from the ellipticity of $3.6 \mu \mathrm{m}$ isophotes. This value could be more appropriate for the innermost part of the source, since the Spitzer data used to infer this is of higher resolution and $\mathrm{HI}$ as a tracer loses potency towards the nuclei of galaxies, as the gas becomes increasingly molecular. As expected, using the lower inclination value from Trujillo et al. resulted in (incrementally) better statistics for both synchrotron and SSC dominated fits, however the overall physical picture did not change. Hence we did not further pursue this avenue, but take this as tentative evidence suggesting the jet inclination in M94* may be less than $29^{\circ}$.

We should note that in our fits the column density $N_{\mathrm{H}}$ was left free to vary in the range noted in Table 2, but consistently pegged at the lower value of $1.41 \times 10^{20} \mathrm{~cm}^{-2}$, reflecting Galactic absorption only. The upper limit of $3.3 \times 10^{20}$ $\mathrm{cm}^{-2}$ mentioned in Table 2 from Eracleous et al. (2002) was obtained by modelling the X-ray spectrum with a simple

${ }^{8}$ In all models the outer disk radius $\mathrm{r}_{\text {out }}$ was fixed to a typical value of $10^{4} r_{\mathrm{g}}$ that avoids fitting any features of the SED with the low-energy part of the disk's thermal spectrum. power-law only model. As our modelling is more complex, and we only use data above $1 \mathrm{keV}$, the upper limit may not be relevant for our modelling. Additionally, in Roberts et al. (1999) it is argued that any additional intrinsic absorption in M94* may be masked by soft thermal emission.

Aside from the difference in energy distribution in terms of magnetic to particle energy densities and acceleration efficiency, the two remaining fits SYN and COM actually reflect a rather similar physical picture as they agree on the remaining parameters within about a factor two. In fact not even the magnetic and particle densities are that different, as the SYN model only just exceeds equipartition while the COM model is not too far below. The electron temperatures, particle distribution indices and jet base-radius-to-height ratio are even in excellent agreement. So although we can not discriminate the dominant radiation mechanism, the underlying physical picture in terms of jet geometry and energy budget are well-represented by these two models, that we will compare below with previous explorations.

\section{DISCUSSION}

Recently Nemmen et al. (2014) also modelled an M94* broadband spectrum. However their modelling was not performed in the context of an outflow-dominated model, but rather focussed on an ADAF-dominant (Advection Dominated Accretion Flow; a type of RIAF) model. In fact, they preclude a jet from being able to fit the M94* X-ray data, noting that the X-ray spectrum would be too hard for a diffusive shock acceleration origin. Their modelling, however, does not include the inverse Compton scattering of seed photons from the jet, while our results clearly indicate that seed photons from an outflow offer an abundant photon reservoir to feed a Compton component. In addition we show that a particle distribution index $p \sim 2.4-2.5$ is adequate to interpret the data. Such values are fully consistent with diffusive shock acceleration (and consistent with Nemmen et al. 2014). Hence this work clearly indicates that not only is a relativistic jet able to explain the observed broadband SED, but also that that a jet model cannot be precluded simply based on the shape of the X-ray spectrum.

\subsection{Comparison with other sources}

Here we compare the M94* model parameters with approximate values and ranges previously obtained within the MNW05 framework, for BHB states as well as for individual AGN. These values and ranges are summarised in Table 4 .

The BHs listed in Table 4 vary considerably in luminosity ( in Eddington units) and the normalised power going into the jets $\left(\mathrm{N}_{\mathrm{j}}\right)$ reflects these luminsities, within about an order of magnitude. This relationship is however not expected to be linear as $\mathrm{N}_{\mathrm{j}}$ also needs to provide e.g. the kinetic proton energy; an unknown. The normalised jet powers we obtained for M94* are very similar to values obtained for M81*, as both M81* and M94* are classifiable as LINERs and they are of similar $L / L_{\text {Edd }}$ luminosity. Interestingly, however, the jet in NGC 4051 is rather more powerful even though NGC 4051 is a Seyfert. As described in Maitra et al. (2011), NGC 4051 may actually be in a transitional state between soft and hard (assuming we can think of AGN in 


\begin{tabular}{|c|c|c|c|c|c|c|c|c|}
\hline \multirow{3}{*}{$\mathrm{N}_{\mathrm{j}}\left(10^{-5} L_{\mathrm{Edd}}\right)$} & \multicolumn{5}{|c|}{$\mathrm{AGN}$} & \multicolumn{3}{|c|}{ BHBs } \\
\hline & NGC 4051 & M81* & \multicolumn{2}{|c|}{ Sgr A* } & M87 & quiescence & HS & $\chi$-state \\
\hline & $2 \times 10^{3}$ & 4 & 0.03 & 0.04 & $0.05-0.2$ & $1-146$ & $30-8400$ & $0.2-2 \times 10^{5}$ \\
\hline $\mathrm{T}_{\mathrm{e}}\left(10^{11} \mathrm{~K}\right)$ & 0.4 & 1 & 3 & 4 & $3-4.5$ & $0.1-0.3$ & $0.3-0.7$ & $0.04-0.09$ \\
\hline $\mathrm{r}_{0}\left(r_{\mathrm{g}}\right)$ & 4 & 5 & 4 & 2 & $3-10$ & $2-4$ & $3-114$ & $4-20$ \\
\hline$k$ & $1^{*}$ & $1-2$ & 1.5 & 0.1 & $0.3-1.1$ & $0.044-2$ & $1.1-5.4$ & $29-707$ \\
\hline $\boldsymbol{\epsilon}_{\text {scat }}\left(10^{-4}\right)$ & - & $5-40$ & $10^{2}$ & $10^{-2}$ & $10^{-3 *}$ & $0.01-25$ & $1-440$ & $0.5-1.7$ \\
\hline$p$ & 2.7 & 2.4 & 2.5 & 2.6 & $2.5-3.0$ & $2.2-3.0$ & $2.2-2.9$ & $1.2-2.5$ \\
\hline$z_{\text {acc }}\left(r_{\mathrm{g}}\right)$ & 5 & $130-200$ & 40 & 23 & $8-50$ & $14-25$ & $7-526$ & $7-844 \times 10^{3}$ \\
\hline$h_{0} / r_{0}$ & 1 & $5-15$ & $1.5^{*}$ & 1.3 & $2-10$ & $1.5^{*}$ & $0.4-1.7$ & $1.5^{*}$ \\
\hline $\log \left(M_{\mathrm{BH}} / M_{\odot}\right)$ & 6.2 & 7.8 & \multirow{2}{*}{\multicolumn{2}{|c|}{$\begin{array}{c}6.6 \\
10^{-4}\end{array}$}} & 9.8 & $\sim 1$ & $\sim 1$ & 1.2 \\
\hline$L_{X}\left(10^{-5} L_{\text {Edd }}\right)$ & $\sim 10^{2}$ & $2-6$ & & & $10^{-2}$ & $<0.1$ & $0.1-10^{3}$ & $\sim 10^{5}$ \\
\hline $\begin{array}{l}\text { Dom. X-ray } \\
\text { rad. mech }\end{array}$ & syn & $\begin{array}{l}\text { syn/ } \\
\text { SSC }\end{array}$ & syn & $\mathrm{SSC}$ & $\mathrm{SSC}$ & $\begin{array}{l}\text { syn/ } \\
\text { SSC }\end{array}$ & $\begin{array}{l}\text { mostly } \\
\text { SSC }\end{array}$ & $\begin{array}{c}\text { SCC } \\
\text { preferred }\end{array}$ \\
\hline References & $\mathrm{a}$ & $\mathrm{b}$ & & & $\mathrm{f}$ & $\mathrm{g}, \mathrm{h}$ & $\mathrm{i}, \mathrm{j}, \mathrm{k}$ & 1 \\
\hline
\end{tabular}

Table 4. Summary of approximate fitted values and parameter ranges for BHBs states and individual AGN from previous MNW05 works. Parameters listed with an asterisk $\left(^{*}\right)$ were frozen to the listed value. Quiescent BBHs are A0620-00 and XTE J1118+480, HS BHBs are GX 339-4, GRO J1655-40 and Cyg X-1, the $\chi$ state BH is GRS 1915+105. For Sgr A* we are quoting results from Connors et al. (2016, in prep.), as these these are the best ones available related to (relatively) high luminosity flares (during which Sgr A* falls on the FP, see text). Also listed are the dominant X-ray radiation mechanism (dom. X-ray rad. mech.) for each AGN/BH state. For Sgr A* values for both synchrotron and SSC dominated fit are shown explicitly. References: a) Maitra et al. (2011), b) Markoff et al. (2008), c) Connors et al. (2016), d)Markoff et al. (2007), e) Maitra et al. (2009b)), f) Prieto et al. (2016), g) Gallo et al. (2007), h) Plotkin et al. (2015), i) MNW05, j) Maitra et al. (2009a)), k) Migliari et al. (2007), l) van Oers et al. (2010).

terms of BHB spectral states). For Sgr A* the difference between $\mathrm{N}_{\mathrm{j}}$ and $L_{\mathrm{X}} / L_{\mathrm{Edd}}$ is rather large. Sgr A* may generally not even be classified as an AGN (e.g. Goldwurm et al. 1994; Mastichiadis \& Ozernoy 1994), due to its extremely low luminosity (e.g. Baganoff et al. 2003). Only in its flaring mode do we observe a non-thermal X-ray spectrum, that increases the X-ray luminosities to fundamental plane values during the brightest flares only (Neilsen et al. 2013; Connors et al. 2016). The $L_{X} / L_{\text {Edd }}$ value we quote in Table 4 is inferred from these non-thermal flares.

At $\sim 10^{11} \mathrm{~K}$, the electron temperatures obtained in both our models are fully consistent with AGN of similar BH mass previously studied within the MNW05 framework as well as with the inner flow of other RIAF models for other LLAGN (e.g. Yuan et al. 2002a,b, 2006; Mościbrodzka et al. 2016). The electron temperatures in the different AGN and BHB states reveal an anti-correlation with the luminosities in Eddington that is qualitatively similar to such correlations found for thermal Comptonisation models (e.g. Miyakawa et al. 2008; Niedźwiecki et al. 2014). There is an indication of a correlation between $\mathrm{T}_{\mathrm{e}}$ and black hole mass, with the BHBs displaying the lowest temperatures and, on the other end of the mass scale, M87 showing the highest mass and the highest electron temperatures. This could be understood if pair production acts as a means for cooling the inner flow (e.g. Begelman et al. 1987), as AGN conditions permit higher electron temperatures before starting pair production. However significant pair production is only expected at high luminosity and high compactness (Malzac et al. 2009).

The M94* fits confirm the trend seen before that within the MNW05 framework, SMBHs and quiescent BHs show a compact acceleration region, with nozzle radii of only a few $r_{\mathrm{g}}$. Similarly, all AGN and quiescent BHBs show equipartition between the magnetic and particle energy densities. The HS BHBs, however, have jets tending towards Poynting flux dominated, with the most luminous BHB GRS 1915+105 displaying the greatest magnetic domination, suggesting a correlation between magnetic domination and normalised jet power for BHBs. Markoff et al. (2008) find evidence for a similar correlation in the case of $\mathrm{M} 81^{*}$ but for the entire AGN sample this evidence is lacking. However we need to bear in mind that this AGN sample is still rather small and modelling was not performed homogeneously. In the future we will be reconsidering all these issues in the context of a new semi-analytical relativistic MHD jet model that is under development, based on work in Polko et al. (2010, 2013, 2014). In summary these trends could be real or they could be artefacts of having too many free parameters at this stage and since the internal pressures are currently decoupled from the jet dynamics. In future work all these parameters will be set up self-consistently.

Interestingly, a more compact jet base together with a sub-equipartition accretion flow (and slightly higher electron temperatures) were also obtained in the SSC dominated fits of the quiescent BHs A0620-00 and XTE J1118+480, when compared to their equivalent synchrotron dominated fits (Gallo et al. 2007; Plotkin et al. 2015, respectively). An equivalent duo of modelling interpretations is seen for Sgr $A^{*}$, where the absolute differences in these three parameters are remarkably similar to those seen in M94*. Unfortunately, for the other very low luminosity LINER 2, M87, only SSC dominated models were presented (Prieto et al. 2016). Judging from the M87 spectra, it appears possible to model this source with synchrotron dominated X-rays. Doing so would provide further insight into the self-similarity witnessed above for the other lowest accretion rate sources. In the higher luminosity M81* similar statistically equivalent SSC/synchrotron model interpretations are not obtained, but there a fundamentally different model is assumed in the SSC dominated fits, where power-law leptons are injected straight into the nozzle. This change in assumptions precludes a direct comparison between the SSC models of M94* and M81*. In any case for the lowest luminosity sources it appears our current modelling can not unequivocally distinguish the dominant radiative mechanism in the X-rays. 
Some works suggest an SSC origin for the lowest Eddington accretion rate sources (Markoff et al. 2001; Plotkin et al. 2015; Prieto et al. 2016), and a synchrotron origin at higher accretion rates $\left(10^{-4} \lesssim L_{\mathrm{X}} / L_{\text {Edd }} \lesssim 10^{-3}\right.$; Russell et al. 2010). In line with these results a XTE J1118+480 transition from the HS to quiescence suggested the preferred model should change from synchrotron to SSC dominated as the Eddington rate decreased (Maitra et al. 2009a; Plotkin et al. 2015). More such evidence comes from the fact that for the highest accretion rate AGN studied with MNW05, the Seyfert NGC 4051, only synchrotron dominated fits were deemed feasible (Maitra et al. 2009a). However this discussion is far from final, particularly considering the Eddington-rate BHB GRS 1915+105 prefers SSC (van Oers et al. 2010).

In terms of the particle distribution index $p$, we see general agreement among all sources, with $2<p<3$. In our current modelling we have included a synchrotron cooling break and adequate fits were found at $p<2.5$, consistent with Heavens \& Drury (1988). Older values of $p>2.5$ from earlier applications of the model were always implicitly assumed to reflect a cooling dominated regime. Therefore it seems likely that if NGC 4051 and M87 (and similarly, high$p$ BHBs) were re-explored with a cooling break inclusive model, reduced values for $p$ would be obtained. However if a break steepens the model spectrum at UV-to-X-ray energies significantly, a different location of the acceleration $z_{\text {acc }}$ is implied. Hence a direct comparison of M94* with previous works, of the optically thick to optically thin turnover (and possible dependencies of it on other parameters), is impossible. For BHBs a correlation between $z_{\text {acc }}$ and $\mathrm{N}_{\mathrm{j}}$ (or, equivalently, and perhaps more importantly, an anti-correlation between mass accretion rate and the break frequency) is seen (Russell et al. 2013). For the LLAGN (excluding the "intermediate state" NGC 4051) we see a similar evolution, however due to the small number of available AGN the evidence is not so clear: The location of the acceleration obtained for M94* are very similar to those found for M81*, especially considering that jets cover up to $10^{7} r_{\mathrm{g}}$ and hence these distances are preferably considered in log-space. Indeed, the fact that all the values in Table 4 fall within $\log \left(z_{\text {acc }}\right) \sim 1-4$ for all black holes of any size is already interesting in itself. Re-modelling of the AGN with a cooling break inclusive model would however be necessary before drawing firm conclusions on any possible relations with $z$ acc. Determining the distance to the acceleration region self-consistently in a jet from theory has been the subject of the work mentioned above by Polko et al. $(2010,2013,2014)$ and is currently under further development (Ceccobello et al. in prep.).

To summarise, the above discussion suggests that the (pure) LINERs previously studied with MNW05 may be closer in nature to quiescent BHBs than to HS BHBs, perhaps unsurprisingly, as these LLAGN display the lowest accretion rates of the AGN class: Compared to their high ionisation counterparts, for a similar galaxy type and stellar mass (that is strongly linked to the central BH mass; Ferrarese \& Merritt 2000; Gebhardt et al. 2000), LINERs typically display nuclear luminosities 1 to 5 orders of magnitude lower (Netzer 2013). However we must bear in mind that the LLAGN discussed here are very similar in Eddington accretion rate to quiescent $\mathrm{BHBs}$, which is a more important factor than the absolute luminosity. In fact, Markoff et al. (2015); Connors et al. (2016) show that when using such mass-invariant accretion rates, MNW05 works well across the entire BH mass scale.

However, as indicated above, from our results there appear to be emerging subtle differences between the AGN and BHB classes, in terms of the level of equipartition and normalised jet power and the dominant radiation mechanism in the X-rays at higher accretion rates. In order to investigate these matters it will certainly help to explore more AGN at the higher end of the accretion rate scale. Only a single NLS1 has been modelled in the context of the MNW05 model up to this point and it would be insightful to see if other radio loud Seyferts would permit SSC or also require synchrotron domination. Furthermore, the AGN and BHB classes show a number of major observational differences: In BHBs, after a hard-to-soft transition (where optically thin relativistic ejecta are launched; e.g. Fender et al. 1999, 2004), no steady jets are observed, only radiatively-driven winds (e.g. Neilsen \& Lee 2009; Ponti et al. 2012). For AGN this is not the case: both slow, steady jets (like those found in the hard state of $\mathrm{XRBs}$ ) and high-power, relativistic jets (like those observed in the hard-to-soft transition in BHBs) can be observed in the radiatively efficient, soft-state-equivalent AGN, sometimes coexisting with winds as well (e.g. Nesvadba et al. 2008; Mullaney et al. 2013; Collet et al. 2016). Although there are various theories, attributing various mechanisms for this observed difference between BHBs and AGN (e.g. Tchekhovskoy et al. 2011; Hardcastle et al. 2007; Sikora \& Begelman 2013; Nemmen \& Tchekhovskoy 2015; Tadhunter 2016), we are far from consensus about the definitive mechanism. Applying the model from MNW05 to other radio-loud Seyferts could help to understand what is causing this difference. Lastly, multi-wavelength quasar SEDs (e.g. Elvis et al. 1994) are necessary to fully explore the higher end of the SMBH accretion range.

Indeed, modelling high accretion rates is not straight forward and other problems will have to be taken into account: First of all we need to account for pair-production in the model. Secondly, at high luminosity the accretion flow is thought to turn efficient, while previously the main objective of MNW05 has been to study the low accretion rate, inefficient accretion flows that fall onto the fundamental plane, as these are unambiguously associated with jets. Although at the highest accretion rates the flow may turn inefficient again, these may be regimes for which an MNW05 jet is not appropriate. But with these caveats, studying higher accretion rate sources and especially "intermediate states" (like GRS 1915+105 and NGC 4051) should be feasible. Such modelling would allow further investigation of the fundamental plane, particularly whether the high accretion rate sources fall onto the appropriate loci extending the efficient track to higher luminosities, or whether they perhaps draw an altogether different radio/X-ray correlation track (e.g. Dong et al. 2014; Xie \& Yuan 2016), possibly providing complementary information on the underlying driving physics and radiation mechanisms.

\section{CONCLUSIONS}

We presented a broadband SED of the nucleus of the closest obscured LINER 2, M94, composed of predominantly sub-arcsec resolution data, and modelled the SED with an 
outflow-dominated model. The results have shown that even though the X-ray spectrum is relatively steep, a jet/diffusive shock acceleration can adequately explain the data. If synchrotron dominated X-rays are assumed, a cooling break is found in that energy regime. With respect to electron temperature, compactness and equipartition in the inner flow our results are completely consistent with previous finding for LLAGN and quiescent BHBs, but unfortunately we also recover and were unable to break commonly found degeneracies, in terms of the dominant radiation mechanisms.

Most importantly however, work done here increases the number of sources that have been explored with jetdominated SEDs, and the growing number allows for increasingly insightful comparisons between different classes of BHs that differ many magnitudes in mass. It is worth noting, however, that especially the high accretion rate regime remains to be investigated in more depth. In this regime different problems such as pair-production, as well as presumably an altogether different type of accretion will require further expansion of the frameworks used. Work in that regard is however well-underway.

Furthermore, as a myriad of new telescope arrays are currently being developed and built, the quality of available SEDs is certain to improve by orders of magnitude, reaching new levels of detail, complexity and signal-to-noise. Moreover the impending transient era (Metzger et al. 2015) is sure to deliver a wealth of new information on jetted sources: As wide field observatories like the Square Kilometer Array, the Cherenkov Telescope Array and the Large Synoptic Survey Telescope will provide large-footprint surveys with a high frequency of repeated observations, yielding accurate time-sampling for BHBs as they go through their outburst and quiescent cycles. Even in the near future improvements can be expected for one of the arrays used in this work: eMERLIN should soon meet its design specifications, sporting a broad $(2 \mathrm{GHz})$ bandwidth at $\mathrm{C}$-band $(5 \mathrm{GHz})$. This will allow us not only to study in detail the short-term radio variability by verifying whether the M94* flat/inverted spectrum is truly as uniform as we see in this work, or whether - because of e.g. absorption - the uniformity breaks down when resolving the radio frequencies into smaller bandwidth pieces (in a way similar to what we observed here in our currently available L-band data), but also to investigate the long-term variability, comparing near-future flux-levels in M94* to those of the oldest data in our data set, namely the data around $5 \mathrm{GHz}$ that are currently over 3 decades old.

Clearly the study presented here would benefit from the addition of (sub)millimetre data. Data from the Atacama Large Millimeter/submillimeter Array, for example, would be extremely beneficial in breaking degeneracies in the modelling, as this would help us in ascertaining the exact energy and shape of one of the most revealing features in our model spectrum; the "sub-mm bump". Considering the importance of this feature in jet physics and the sensitivity of this feature on other jet parameters, having this data available would surely allow us to preclude a number of interpretations as viable solutions. In addition, sub-mm and $\mathrm{mm}$ wavelengths have the added benefit that they offer the highest angular resolution in this band. In particular, most LLAGN turn optically thin and are more transparent at shorter wavelength, starting in the (sub)mm band. VLBI at these higher frequencies would allow observation of the core regions of the accretion zone that are hidden from view at lower radio frequencies, ultimately even allowing direct imaging of the central engine of AGN. Although it will not be able to resolve the core in M94, the submm observations the Event Horizon Telescope (Honma et al. 2016) will make when it comes online in a few years are sure to provide a wealth of new insight into the nature of accretion flows, jets and AGN, as it should for the first time be able to glimpse what exactly is happening near the edge of a $\mathrm{BH}$.

In the meantime any authoritative model would need to tackle issues of degeneracy and deal with these issues with a self consistent approach. The ground work for this has been laid in Polko et al. (2010, 2013, 2014). However until implementations of these papers are complete (Ceccobello et al. in prep.), individual source studies with currently available frameworks - as done here - will help discern trends via comparative studies and pinpoint the relevant parameter space for this next generation of all-encompassing fully self-consistent magnetohydrodynamic models.

\section{ACKNOWLEDGEMENTS}

We thank Pierre-Emmanuel Belles and Rob Beswick for assistance reducing the e-MERLIN radio images. We thank the referee and Thomas Russell for useful comments. The NVAS images used were produced as part of the NRAO VLA Archive Survey, (c) AUI/NRAO. The National Radio Astronomy Observatory is a facility of the National Science Foundation operated under cooperative agreement by Associated Universities, Inc.

\section{REFERENCES}

Arnaud K. A., 1996, in Jacoby G. H., Barnes J., eds, Astronomical Society of the Pacific Conference Series Vol. 101, Astronomical Data Analysis Software and Systems V. pp 17-+

Asmus D., Hönig S. F., Gandhi P., Smette A., Duschl W. J., 2014, MNRAS, 439, 1648

Baganoff F. K., et al., 2003, ApJ, 591, 891

Baldwin J. A., Phillips M. M., Terlevich R., 1981, PASP, 93, 5

Begelman M. C., Sikora M., Rees M. J., 1987, ApJ, 313, 689

Belloni T. M., 2010, in Belloni T., ed., Lecture Notes in Physics, Berlin Springer Verlag Vol. 794, Lecture Notes in Physics, Berlin Springer Verlag. p. 53 (arXiv:0909.2474), doi:10.1007/978-3-540-76937-8_3

Beloborodov A. M., 1999, ApJ, 510, L123

Biretta J. A., Stern C. P., Harris D. E., 1991, AJ, 101, 1632

Bîrzan L., Rafferty D. A., McNamara B. R., Wise M. W., Nulsen P. E. J., 2004, ApJ, 607, 800

Blandford R. D., Konigl A., 1979, ApJ, 232, 34

Bruzual G., Charlot S., 2003, MNRAS, 344, 1000

Buta R. J., Corwin H. G., Odewahn S. C., 2007, The de Vaucouleurs Altlas of Galaxies. Cambridge University Press

Calzetti D., Kinney A. L., Storchi-Bergmann T., 1994, ApJ, 429, 582

Carilli C. L., Perley R. A., Dreher J. W., Leahy J. P., 1991, ApJ, 383,554

Ceccobello et al. C., "in prep.", MNRAS

Clemens M. S., Vega O., Bressan A., Granato G. L., Silva L., Panuzzo P., 2008, A\&A, 477, 95

Collet C., et al., 2016, A\&A, 586, A152

Condon J. J., 1992, ARA\&A, 30, 575 
Connors R. M. T., et al., 2016, preprint, (arXiv:1612.00953)

Constantin A., Seth A. C., 2012, Advances in Astronomy, 2012, 13

Doane N. E., Sanders W. T., Wilcots E. M., Juda M., 2004, AJ, 128,2712

Dong A.-J., Wu Q., Cao X.-F., 2014, ApJ, 787, L20

Donovan Meyer J., et al., 2013, ApJ, 772, 107

Dopita M. A., et al., 2005, ApJ, 619, 755

Elvis M., et al., 1994, ApJS, 95, 1

Eracleous M., Shields J. C., Chartas G., Moran E. C., 2002, ApJ, 565,108

Eracleous M., Hwang J. A., Flohic H. M. L. G., 2010, ApJS, 187, 135

Erwin P., 2004, A\&A, 415, 941

Esin A. A., McClintock J. E., Narayan R., 1997, ApJ, 489, 865

Falcke H., 1996, ApJ, 464, L67+

Falcke H., Körding E., Markoff S., 2004, A\&A, 414, 895

Fender R. P., Garrington S. T., McKay D. J., Muxlow T. W. B., Pooley G. G., Spencer R. E., Stirling A. M., Waltman E. B., 1999, MNRAS, 304, 865

Fender R. P., Belloni T. M., Gallo E., 2004, MNRAS, 355, 1105

Fernández-Ontiveros J. A., Prieto M. A., Acosta-Pulido J. A., Montes M., 2012, Journal of Physics Conference Series, 372, 012006

Ferrarese L., Merritt D., 2000, ApJ, 539, L9

Fruscione A., et al., 2006, in Society of Photo-Optical Instrumentation Engineers (SPIE) Conference Series. p. 1, doi:10.1117/12.671760

Gallo E., Migliari S., Markoff S., Tomsick J. A., Bailyn C. D., Berta S., Fender R., Miller-Jones J. C. A., 2007, ApJ, 670, 600

Garrington S. T., et al., 2004, in Oschmann Jr. J. M., ed., Proc. SPIEVol. 5489, Ground-based Telescopes. pp 332-343, doi: $10.1117 / 12.553235$

Gebhardt K., et al., 2000, ApJ, 539, L13

Gilfanov M., 2010, in Belloni T., ed., Lecture Notes in Physics, Berlin Springer Verlag Vol. 794, Lecture Notes in Physics, Berlin Springer Verlag. p. 17 (arXiv:0909.2567), doi:10.1007/978-3-540-76937-8_2

Gillessen S., Eisenhauer F., Fritz T. K., Bartko H., Dodds-Eden K., Pfuhl O., Ott T., Genzel R., 2009, ApJ, 707, L114

Goldwurm A., et al., 1994, Nature, 371, 589

Greisen E. W., 2003, Information Handling in Astronomy - Historical Vistas, 285, 109

Hardcastle M. J., Evans D. A., Croston J. H., 2007, MNRAS, 376, 1849

Heavens A. F., Drury L. O., 1988, MNRAS, 235, 997

Ho L. C., 1999, ApJ, 516, 672

Ho L. C., Peng C. Y., 2001, ApJ, 555, 650

Ho L. C., Filippenko A. V., Sargent W. L. W., 1997, ApJS, 112, 315

Honma M., Akiyama K., Tazaki F., Kuramochi K., Ikeda S., Hada K., Uemura M., 2016, Journal of Physics Conference Series, 699, 012006

Houck J. C., Denicola L. A., 2000, in Manset N., Veillet C., Crabtree D., eds, Astronomical Society of the Pacific Conference Series Vol. 216, Astronomical Data Analysis Software and Systems IX. pp 591-+

Hubble E. P., 1926, ApJ, 64, 321

Jensen J. B., Tonry J. L., Barris B. J., Thompson R. I., Liu M. C., Rieke M. J., Ajhar E. A., Blakeslee J. P., 2003, ApJ, 583, 712

Jokipii J. R., 1987, ApJ, 313, 842

Kardashev N. S., 1962, Soviet Astronomy, 6, 317

Kauffmann G., et al., 2003, MNRAS, 346, 1055

Keel W. C., et al., 2012, MNRAS, 420, 878

Keel W. C., et al., 2015, AJ, 149, 155

Kewley L. J., Groves B., Kauffmann G., Heckman T., 2006, MNRAS, 372,961
Kinney A. L., Schmitt H. R., Clarke C. J., Pringle J. E., Ulvestad J. S., Antonucci R. R. J., 2000, ApJ, 537, 152

Koay J. Y., Vestergaard M., Bignall H. E., Reynolds C., Peterson B. M., 2016, preprint, (arXiv:1602.07289)

Körding E., Colbert E., Falcke H., 2005, A\&A, 436, 427

Körding E. G., Jester S., Fender R., 2006, MNRAS, 372, 1366

Kormendy J., Ho L. C., 2013, ARA\&A, 51, 511

LaMassa S. M., et al., 2015, ApJ, 800, 144

Lemoine M., Pelletier G., 2003, ApJ, 589, L73

Maitra D., Markoff S., Brocksopp C., Noble M., Nowak M., Wilms J., 2009a, MNRAS, 398, 1638

Maitra D., Markoff S., Falcke H., 2009b, A\&A, 508

Maitra D., Miller J. M., Markoff S., King A., 2011, ApJ, 735, 107

Makishima K., Maejima Y., Mitsuda K., Bradt H. V., Remillard R. A., Tuohy I. R., Hoshi R., Nakagawa M., 1986, ApJ, 308, 635

Malzac J., Beloborodov A. M., Poutanen J., 2001, MNRAS, 326, 417

Malzac J., Belmont R., Fabian A. C., 2009, MNRAS, pp 1453-+

Maoz D., Filippenko A. V., Ho L. C., Rix H.-W., Bahcall J. N., Schneider D. P., Macchetto F. D., 1995, ApJ, 440, 91

Maoz D., Nagar N. M., Falcke H., Wilson A. S., 2005, ApJ, 625, 699

Markoff S., Falcke H., Yuan F., Biermann P. L., 2001, A\&A, 379, L13

Markoff S., Nowak M. A., Wilms J., 2005, ApJ, 635, 1203

Markoff S., Bower G. C., Falcke H., 2007, MNRAS, 379, 1519

Markoff S., et al., 2008, ApJ, 681, 905

Markoff S., et al., 2015, ApJ, 812, L25

Mastichiadis A., Ozernoy L. M., 1994, ApJ, 426, 599

McHardy I. M., Koerding E., Knigge C., Uttley P., Fender R. P., 2006, Nature, 444, 730

Merloni A., Fabian A. C., 2002, MNRAS, 332, 165

Merloni A., Heinz S., di Matteo T., 2003, MNRAS, 345, 1057

Metzger B. D., Williams P. K. G., Berger E., 2015, ApJ, 806, 224

Migliari S., et al., 2007, ApJ, 670, 610

Mitsuda K., et al., 1984, PASJ, 36, 741

Miyakawa T., Yamaoka K., Homan J., Saito K., Dotani T., Yoshida A., Inoue H., 2008, PASJ, 60, 637

Mościbrodzka M., Falcke H., Shiokawa H., 2016, A\&A, 586, A38

Moustakas J., Kennicutt Jr. R. C., 2006, ApJS, 164, 81

Mullaney J. R., Alexander D. M., Fine S., Goulding A. D., Harrison C. M., Hickox R. C., 2013, MNRAS, 433, 622

Nagar N. M., Falcke H., Wilson A. S., Ulvestad J. S., 2002, A\&A, 392,53

Nagar N. M., Falcke H., Wilson A. S., 2005, A\&A, 435, 521

Neilsen J., Lee J. C., 2009, Nature, 458, 481

Neilsen J., et al., 2013, ApJ, 774, 42

Nemmen R. S., Tchekhovskoy A., 2015, MNRAS, 449, 316

Nemmen R. S., Storchi-Bergmann T., Eracleous M., 2014, MNRAS, 438, 2804

Nesvadba N. P. H., Lehnert M. D., De Breuck C., Gilbert A. M., van Breugel W., 2008, A\&A, 491, 407

Netzer H., 2013, The Physics and Evolution of Active Galactic Nuclei

Netzer H., 2015, preprint, (arXiv:1505.00811)

Niedźwiecki A., Xie F.-G., Stępnik A., 2014, MNRAS, 443, 1733

Novikov I. D., Thorne K. S., 1973, in Dewitt C., Dewitt B. S., eds, Black Holes (Les Astres Occlus). pp 343-450

Pellegrini S., Fabbiano G., Fiore F., Trinchieri G., Antonelli A., 2002, A\&A, 383, 1

Pian E., Romano P., Maoz D., Cucchiara A., Pagani C., Parola V. L., 2010, MNRAS, 401, 677

Plotkin R. M., Markoff S., Kelly B. C., Körding E., Anderson S. F., 2012, MNRAS, 419, 267

Plotkin R. M., Gallo E., Markoff S., Homan J., Jonker P. G., Miller-Jones J. C. A., Russell D. M., Drappeau S., 2015, MNRAS, 446, 4098 
Polko P., Meier D. L., Markoff S., 2010, ApJ, 723, 1343

Polko P., Meier D. L., Markoff S., 2013, MNRAS, 428, 587

Polko P., Meier D. L., Markoff S., 2014, MNRAS, 438, 959

Ponti G., Fender R. P., Begelman M. C., Dunn R. J. H., Neilsen J., Coriat M., 2012, MNRAS, 422, 11

Prieto M. A., Fernández-Ontiveros J. A., Markoff S., Espada D., González-Martín O., 2016, MNRAS, 457, 3801

Remillard R. A., McClintock J. E., 2006, ARA\&A, 44, 49

Richings A. J., Uttley P., Körding E., 2011, MNRAS, 415, 2158

Roberts T. P., Warwick R. S., Ohashi T., 1999, MNRAS, 304, 52

Roberts T. P., Schurch N. J., Warwick R. S., 2001, MNRAS, 324, 737

Russell D. M., Maitra D., Dunn R. J. H., Markoff S., 2010, MNRAS, 405, 1759

Russell D. M., et al., 2013, MNRAS, 429, 815

Schawinski K., Thomas D., Sarzi M., Maraston C., Kaviraj S., Joo S.-J., Yi S. K., Silk J., 2007, MNRAS, 382, 1415

Schawinski K., et al., 2010, ApJ, 724, L30

Schawinski K., Koss M., Berney S., Sartori L. F., 2015, MNRAS, 451,2517

Seaton M. J., 1979, MNRAS, 187, 73P

Seyfert C. K., 1943, ApJ, 97, 28

Shakura N. I., Sunyaev R. A., 1973, A\&A, 24, 337

Sikora M., Begelman M. C., 2013, ApJ, 764, L24

Stark A. A., Gammie C. F., Wilson R. W., Bally J., Linke R. A., Heiles C., Hurwitz M., 1992, ApJS, 79, 77

Sunyaev R. A., Truemper J., 1979, Nature, 279, 506

Tadhunter C., 2016, A\&A Rev., 24, 10

Tchekhovskoy A., Narayan R., McKinney J. C., 2011, MNRAS, 418, L79

Thorne K. S., Price R. H., 1975, ApJ, 195, L101

Tonry J. L., Dressler A., Blakeslee J. P., Ajhar E. A., Fletcher A. B., Luppino G. A., Metzger M. R., Moore C. B., 2001, ApJ, 546, 681

Trujillo I., Martinez-Valpuesta I., Martínez-Delgado D., Peñarrubia J., Gabany R. J., Pohlen M., 2009, ApJ, 704, 618

Ulvestad J. S., Wilson A. S., 1989, ApJ, 343, 659

Veilleux S., Osterbrock D. E., 1987, ApJS, 63, 295

Xie F.-G., Yuan F., 2016, MNRAS, 456, 4377

Younes G., Porquet D., Sabra B., Reeves J. N., 2011, A\&A, 530, A149

Yuan F., Markoff S., Falcke H., 2002a, A\&A, 383, 854

Yuan F., Markoff S., Falcke H., Biermann P. L., 2002b, A\&A, 391, 139

Yuan F., Taam R. E., Xue Y., Cui W., 2006, ApJ, 636, 46

de Blok W. J. G., Walter F., Brinks E., Trachternach C., Oh S.-H., Kennicutt Jr. R. C., 2008, AJ, 136, 2648

van Oers P., et al., 2010, MNRAS, 409, 763

van der Laan T. P. R., et al., 2015, A\&A, 575, A83

APPENDIX A: e-MERLIN CONTOUR PLOTS 

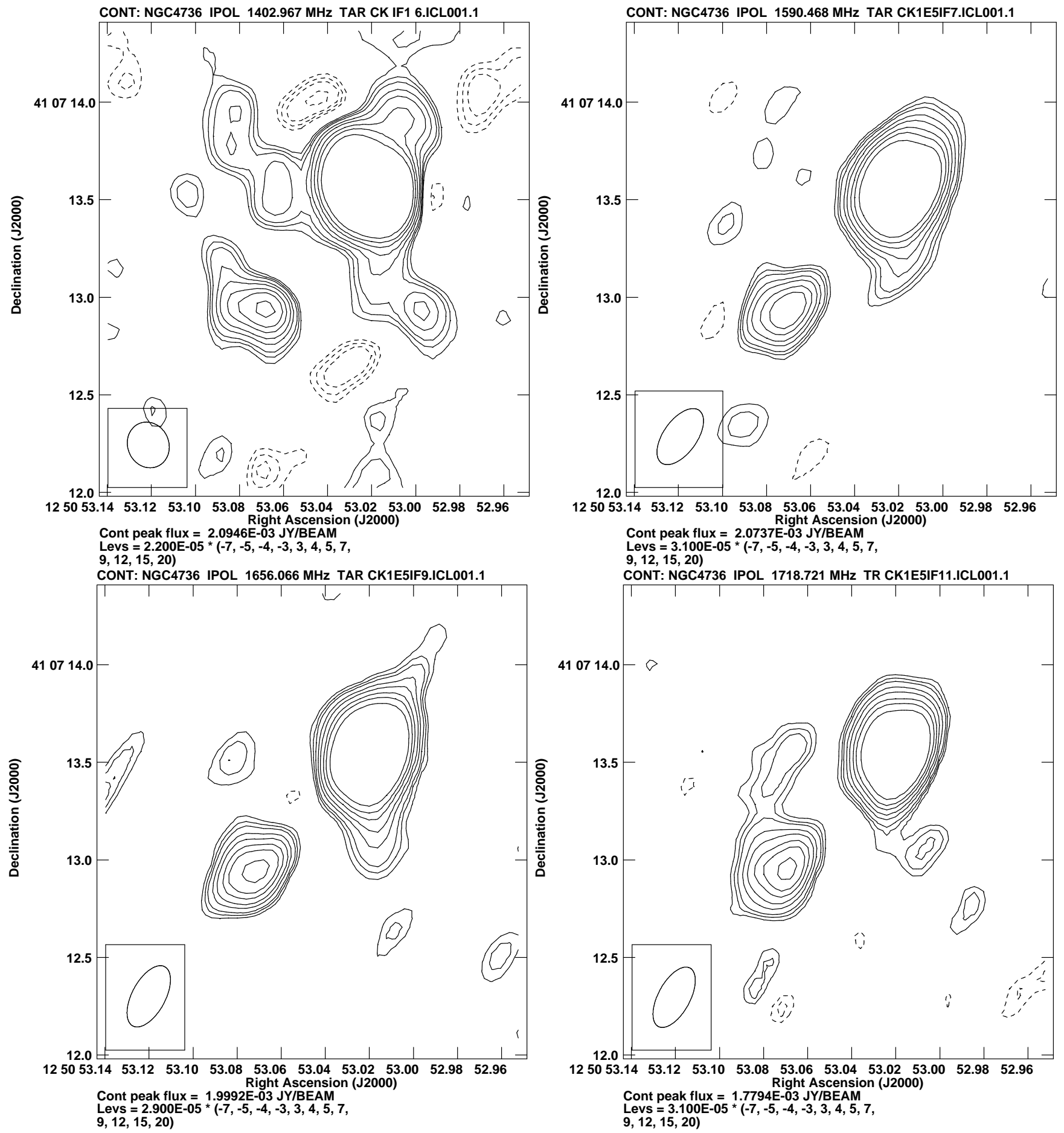

Figure A.1. Contour plots of SPWs 1 through 6 binned together (top left) and of SPW 7 (top right), 9 (bottom left) and 11 (bottom right) individually. Contour levels are plotted as specified in the image. 